\title{
可用带宽度量系统中的若干基本问题
}

周 辉 1,4 , 李 丹 $^{2}$, 王永吉 $1,3+$

${ }^{1}$ (中国科学院 软件研究所, 北京 100190)

${ }^{2}$ (清华大学 计算机科学与技术系,北京 100084)

${ }^{3}$ (中国科学院 计算机科学国家重点实验室, 北京 100190)

${ }^{4}$ (中国科学院 研究生院, 北京 100049)

\section{Fundamental Problems with Available Bandwidth Measurement Systems}

\author{
ZHOU Hui ${ }^{1,4}$, LI Dan ${ }^{2}, \quad$ WANG Yong-Ji ${ }^{1,3+}$ \\ ${ }^{1}$ (Institute of Software, The Chinese Academy of Sciences, Beijing 100190, China) \\ ${ }^{2}$ (Department of Computer Science and Technology, Tsinghua University, Beijing 100084, China) \\ ${ }^{3}$ (State Key Laboratory of Computer Science, The Chinese Academy of Sciences, Beijing 100190, China) \\ ${ }^{4}$ (Graduate University, The Chinese Academy of Sciences, Beijing 100049, China) \\ + Corresponding author: E-mail: ywang@itechs.iscas.ac.cn, http://www.ios.cn/
}

Zhou H, Li D, Wang YJ. Fundamental problems with available bandwidth measurement systems. Journal of Software, 2008,19(5):1234-1255. http://www.jos.org.cn/1000-9825/19/1234.htm

Abstract: Based on the analysis of 13 well-known available bandwidth systems and the experience in designing, building, and deploying the BNeck system, the fundamental problems with available bandwidth measurement systems are analyzed comprehensively.

Key words: computer network; available bandwidth; network measurement; active probing

摘 要: 结合过去 3 年中设计、开发和部署 BNeck 系统的经验, 分析了 13 个可用带宽度量系统的工作原理及软 件实现, 并在此基础上总结了可用带宽度量系统中存在的若干基本问题.对这些问题的研究, 不仅有利于设计和开发 出高效、准确的度量系统,也有利于推动网络管理和视频通信等多个相关领域的共同发展.

关键词: 计算机网络;可用带宽;网络度量;主动探测

中图法分类号: TP393 文献标识码: A

可用带宽是指在不影响网络路径中背景数据流的情况下,端到端通信所能获得的最大数据传输率 ${ }^{[1]}$.可用 带宽是计算机网络的核心概念之一,它在协议设计、网络管理、QoS 部署、组播通信和多媒体传输等诸多方面

\footnotetext{
* Supported by the National Natural Science Foundation of China under Grant Nos.60673022, 60273026, 60473060 (国家自然科学基 金); the National High-Tech Research and Development Plan of China under Grant Nos.2004AA1Z2100, 2005AA113140 (国家高技术研 究发展计划(863)); the One-Hundred-People Program of the Chinese Academy of Sciences under Grant No.BCK35873 (中国科学院百人 计划); the Chinese Academy of Sciences and the Royal Society of the United Kingdom Project under Grant Nos.20030389, 20032006 (中 国科学院与英国皇家学会联合资助项目)

Received 2006-09-12; Accepted 2007-01-23
} 
有着重要意义.例如,TCP 协议的一个主要设计目标就是在不堵塞网络的情况下,最大限度地利用可用带宽 ${ }^{[2]}$.在 每一个网络中, 管理员都需要监测各个链路的负载情况进而合理地配置网络资源, 可用带宽正是网络监测和配 置的重要参考依据. 而且, 能否获得预期的可用带宽, 也是网络运营商(ISP)部署 QoS 的关键考核指标. 与此同时, 大量的网络应用也迫切需要具备快速度量可用带宽的能力.组播通信就需要在占用最小网络资源(包括可用带 宽)的情况下,协同多个网络节点以维护高效的组播路由树 ${ }^{[3]}$. 多媒体应用如点对点视频会议, 同样需要快速感知 各节点间可用带宽的变化,并据此及时调整数据压缩算法和传输策略.

在过去的 10 年里,为了快速、准确地度量可用带宽,国内外学者们基于主动探测机制研发了多个度量系统. 以这些度量系统为主的设计、开发、实验、评估及相关的研究工作极大地推动了网络度量技术的发展. 然而到 目前为止, 这些度量系统仍然处于实验研究的阶段,始终没有一个系统能够满足广域网环境中的度量要求 ${ }^{[4]}$, 更 没有一个系统被互联网用户广泛接受和推广.为此,迫切需要我们去研究度量系统中普遍存在的基本问题,找到 限制系统适用范围的根本原因.

本文结合过去 3 年中设计、开发和部署 BNeck 系统 ${ }^{[5]}$ 的经验,分析了 13 个常见度量系统的工作原理及软 件实现, 同时在不同软、硬件配置的单个节点内、机构内部搭建的实验网络中以及跨地域的覆盖型实验网络上, 针对各种应用场景开展了大规模的实验,进而在理论分析和实验数据的基础上总结了可用带宽度量系统中普 遍存在的基本问题.分析表明,正是这些基本问题极大地限制了度量系统的适用范围.深入研究这些问题,不仅 有利于设计和开发出在复杂网络环境中高效运行的度量系统,而且有利于从整体上推动网络度量技术的发展. 下文首先介绍可用带宽的基本定义,然后根据运行模式将度量系统分为单终端系统和双终端系统两类,并讨论 它们的工作原理和假设前提. 接下来从计时误差、低接入带宽和数据包异常等不同角度研究两类度量系统中共 同存在的基本问题,然后,根据单、双终端系统各自的特点,进一步讨论两类系统中分别存在的基本问题.

\section{1 可用带宽及其度量}

\section{1 基本定义}

可用带宽是在网络路径的上下文中定义的.网络路径由一系列存储转发链路组成, 它能够把数据包从源终 端主机 $\left(R_{0}\right)$ 通过一系列中间节点 $R_{1}, R_{2}, \ldots, R_{n-1}$ 传输到目标终端主机 $\left(R_{n}\right)$. 链路 $L_{i}$ 是从 $R_{i}$ 到 $R_{i+1}$ 的数据传输通道, 这里, $0 \leq i \leq n-1$. 在 $t$ 时刻, $L_{i}$ 的利用率 $U_{i}(t)$ 为

$$
U_{i}(t)= \begin{cases}0, & \text { 当 } L_{i} \text { 空闲时 } \\ 1, & \text { 当 } L_{i} \text { 传输数据包时 }\end{cases}
$$

当 $U_{i}(t)=0$ 时, $L_{i}$ 的传输速率为 0 ; 反之, $L_{i}$ 以固定的速率 $C_{i}$ 传输数据包. 这里, $C_{i}$ 又称为 $L_{i}$ 的物理传输速率或 带宽容量(capacity), 它是由链路的物理特性决定的, 与时间 $t$ 无关. 端到端 $\left(R_{0}\right.$ 到 $\left.R_{n}\right)$ 数据传输率的上限 $C_{P}$ 由最小 的链路带宽容量决定,

$$
C_{P}=\min _{i=0 . . n-1}\left\{C_{i}\right\}
$$

决定 $C_{P}$ 的那段链路称为狭窄链路(narrow link $)^{[1]}$. 同一网络路径中可能存在多条狭窄链路.

在某一时段 $[t, t+\tau]$ 内, 链路 $L_{i}$ 的利用率为

$$
U_{i}(t, t+\tau)=\frac{1}{\tau} \int_{t}^{t+\tau} U_{i}(t) \mathrm{d} t
$$

易知 $U_{i}(t, t+\tau) \in[0,1]$. 在时段 $[t, t+\tau]$ 内, $L_{i}$ 的平均数据传输率为

$$
\lambda_{i}=C_{i} \cdot U_{i}(t, t+\tau)
$$

一般称 $\lambda_{i}$ 为 $L_{i}$ 中背景数据流(cross-traffic)的传输速率. 背景数据流即 $L_{i}$ 正常传输的数据流, 这样称呼是为了 把它们与度量系统出于探测目的而发送的探测数据流(probe-traffic)区分开来.

在时段 $[t, t+\tau]$ 内, $L_{i}$ 的可用带宽 $A_{i}$ 是指 $L_{i}$ 的平均空闲传输速率,即

$$
A_{i}(t, t+\tau)=\frac{1}{\tau} \int_{t}^{t+\tau}\left(C_{i}-\lambda_{i}(t)\right) \mathrm{d} t
$$


本文所关注的可用带宽并非单个链路的可用带宽, 而是待测网络路径的端到端可用带宽 $A_{P}$, 它由路径中最 小的链路可用带宽决定:

$$
A_{P}(t, t+\tau)=\min _{i=0 . . n-1}\left\{A_{i}(t, t+\tau)\right\}
$$

决定 $A_{P}$ 的那段链路常被称为紧凑链路(tight link) ${ }^{[6]}$ 或瓶颈链路(bottleneck link) ${ }^{[5]}$. 与狭窄链路不同, 在一个 网络路径中,瓶颈链路往往只有一条,且其位置还会随着网络负载的不同而发生变化.

在实际度量过程中, 时间 $\tau$ 并不是固定的值, 按照惯例, 它等于度量系统的一个度量周期(即运行一次系统以 度量 $A_{P}$ 所用的时间 $)^{[1]}$. 所以,对于不同的度量系统而言, 时间 $\tau$ 通常不同; 即使是同一个系统, 在软、硬件配置或者 待测路径的属性发生变化时, $\tau$ 也会随之变化.

图 1 是上述概念的简单示例,其中, $R_{0}$ 和 $R_{n}$ 作为源终端主机和目标终端主机分别位于路径的首、尾两端, 链路 $L_{i}$ 的可用带宽 $A_{i}$ 等于带宽容量 $C_{i}$ 减去背景数据流的传输速率 $\lambda_{i}$. 对于一个中间节点而言, 背景数据流可以 有多个来源(source)和去处(sink).

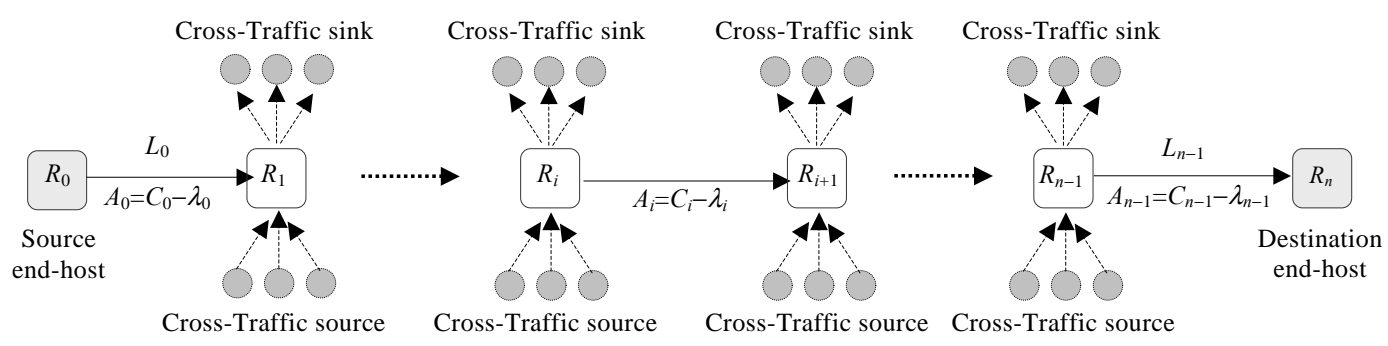

Fig.1 A simplified model of end-to-end network path

图 1 端到端网络路径的简化模型

\section{2 度量系统}

在过去的 10 年里,为了快速而准确地度量可用带宽,研究者们基于主动探测机制研发了大量的软件系统.

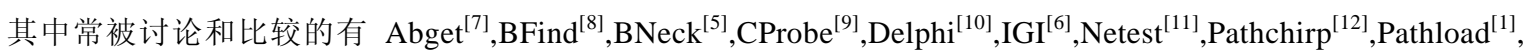
Pipechar ${ }^{[11]}$, SProbe $^{[13]}$, Spruce ${ }^{[14]}$ 和 TOPP ${ }^{[15]}$ 这 13 个系统(见表 1).按照运行模式的不同,这些系统可以分为单终端 系统和双终端系统两类. 其主要区别是, 单终端系统仅需在 $R_{0}$ 上安装软件, 而双终端系统要求在 $R_{0}$ 和 $R_{n}$ 上都安 装. 相比之下, 双终端系统的度量结果更准确, 这是因为它能够利用 $R_{0}$ 和 $R_{n}$ 的协作简化度量过程, 而不像单终端 系统那样必须面对反向路径 $\left(R_{n}\right.$ 到 $\left.R_{0}\right)$ 的干扰. 但是, 双终端系统的应用范围非常有限, 其原因是用户往往只能在 $R_{0}$ (一般是用户自己的机器)上安装软件, 而没有在远程的 $R_{n}$ 上安装软件的权限. 与此相反, 单终端系统只需要安 装在 $R_{0}$ 上, 就能用来度量以 $R_{0}$ 为源终端主机的绝大多数网络路径的可用带宽.

第一个单终端系统是 CProbe ${ }^{[9]}$, 它同时也是最早的可用带宽度量系统.CProbe 充分利用了 ICMP 协议的请 求-应答机制 ${ }^{[16]}$. 具体地,CProbe 从 $R_{0}$ 向 $R_{n}$ 发送多组用于探测的 IP 包(IP 包中的 time-to-live 域被设置为 $1, n$ 之 间的值,且 destination port 域设置成一个非监听端口); 这些 IP 包触发了节点的 ICMP 错误处理流程, 所以, $R_{1}, \ldots, R_{n}$ 会向 $R_{0}$ 发送 ICMP time-exceeded (TE)和 destination-unreachable (DU)包;CProbe 接收到 ICMP TE 和 DU 包后,将根据接收时间推算探测 IP 包在每段链路上传输速率的变化情况,进而估算可用带宽. 在 CProbe 之后, Pipechar ${ }^{[11]}$ 也采用了基于 ICMP 协议的方法, 而 SProbe ${ }^{[13]}$ 则是基于 TCP 和 HTTP 协议开发的, 它们都有目的地 利用了协议的特性以获取远程节点的反馈,并挖掘应答数据包的间隔变化规律进而估算可用带宽.但文献[17] 中的工作证明了 CProbe 和 Pipechar 所度量的其实并不是可用带宽, 而是处于可用带宽 $A_{P}$ 和带宽容量 $C_{P}$ 之间 的非对称分散速率(asymptotic dispersion rate). 所以,在 CProbe 和 Pipechar 之后的单终端系统, 都必须证明它们 所度量的确实是可用带宽.在这些后续的系统中, 常被讨论的有 Abget $^{[7]}$, BFind $^{[8]}$ 和 BNeck ${ }^{[5]}$.

最典型的双终端系统是 Pathload ${ }^{[1]}$.Pathload 的程序分为 pathload_snd 和 pathload_rcv 两部分, 其中, pathload_snd 安装在 $R_{0}$ 上, 而 pathload_rcv 安装在 $R_{n}$ 上.初始状态下,Pathload认为可用带宽属于区间 $\left[0, C_{0}\right]$. 然后, 
pathload_snd 以速率 $x=C_{0} / 2$ 发送 $y$ 组探测数据包(默认情况下 $\left.y=6\right) \cdot R_{n}$ 上的 pathload_rcv 在接收到每一组数据 包后, 计算相邻探测数据包的间隔是否呈递增趋势. 如果多组数据包的间隔都呈递增趋势, 那么,Pathload 认为 $x>A_{P}$ 且 $A_{P} \in[0, x]$; 否则, $x \leq A_{P}$ 且 $A_{P} \in\left[x, C_{0}\right]$. 假设在一次探测完毕后 $A_{P} \in[a, b]$, 那么 pathload_snd 会以 $x=(a+b) / 2$ 的 速率发送 $y$ 组数据包再一次探测网络路径并缩小可用带宽的所属区间. 当 $(b-a)<\sigma$ (或出现了意外)时,Pathload才 停止探测并输出 $[a, b]$, 这里, $\sigma$ 是事先设定的阈值. 需要注意的是,Pathload 最终输出的是一个带宽区间, 而不是单 一数值.除了 Pathload 以外, 常见的双终端系统还有 Delphi ${ }^{[10]}, \mathrm{IGI}^{[6]}$, Netest $^{[11]}$, Pathchirp $^{[12]}{ }^{[12}$ Spruce $^{[14]}$ 和 TOPP ${ }^{[15]}$.

Table 1 Measurement systems

表 1 度量系统

\begin{tabular}{|c|c|c|c|c|c|}
\hline Type & System & Developer \& affiliation & Used protocol $^{*}$ & Technical feature & Year \\
\hline \multirow{6}{*}{$\begin{array}{l}\text { Single } \\
\text { end-host } \\
\text { system }\end{array}$} & Abget & $\begin{array}{l}\text { Antoniades, et al., } \\
\text { ICS, Greece }\end{array}$ & ТСР, НTTP & $\begin{array}{l}\text { Generates fake ACK packets to hack TCP } \\
\text { connection }\end{array}$ & 2005 \\
\hline & BFind & $\begin{array}{c}\text { Akella, et al., } \\
\text { Carnegie-Mellon Univ. } \\
\text { and IBM Research }\end{array}$ & UDP, ICMP & $\begin{array}{l}\text { Uses traceroute to monitor the queue size of } \\
\text { middle nodes, linearly increases probe rate }\end{array}$ & 2002 \\
\hline & BNeck & $\begin{array}{c}\text { Zhou, et al., } \\
\text { Institute of Software }\end{array}$ & UDP, ICMP & $\begin{array}{l}\text { Gets the location, available-bw and capacity } \\
\text { of bottleneck in one measurement cycle }\end{array}$ & 2003 \\
\hline & Cprobe & $\begin{array}{l}\text { Carter and Crovella, } \\
\text { Boston Univ. }\end{array}$ & ICMP & $\begin{array}{l}\text { Combines } \text { Bprobe }^{[9]} \text {, direct available-bw } \\
\text { computation }\end{array}$ & 1996 \\
\hline & Pipechar & $\begin{array}{c}\text { Jin, et al., } \\
\text { UC Berkeley }\end{array}$ & ICMP & $\begin{array}{l}\text { Packet pair technique, used in } \mathrm{NCS}^{[11]} \\
\text { infrastructure }\end{array}$ & 2001 \\
\hline & SProbe & $\begin{array}{c}\text { Saroiu, et al., } \\
\text { Univ. of Washington }\end{array}$ & ТСР, НТTP & $\begin{array}{l}\text { Uses TCP protocol features to get designed } \\
\text { TCP data }\end{array}$ & 2002 \\
\hline \multirow{7}{*}{$\begin{array}{l}\text { Double } \\
\text { end-host } \\
\text { system }\end{array}$} & Delphi & $\begin{array}{l}\text { Ribeiro, et al., } \\
\text { Rice Univ. }\end{array}$ & UDP & $\begin{array}{l}\text { Multifractal cross-traffic, link utilization } \\
\text { estimation }\end{array}$ & 2000 \\
\hline & IGI & $\begin{array}{c}\text { Hu and Peter, } \\
\text { Carnegie-Mellon Univ. }\end{array}$ & UDP & $\begin{array}{l}\text { Exploits the relation between initial gap and } \\
\text { bottleneck gap }\end{array}$ & 2003 \\
\hline & Netest & $\begin{array}{l}\text { Jin and Tierney, } \\
\text { UC Berkeley }\end{array}$ & UDP & Monitors maximum burst size & 2002 \\
\hline & Pathchirp & $\begin{array}{l}\text { Ribeiro, et al., } \\
\text { Rice Univ. }\end{array}$ & UDP & $\begin{array}{l}\text { Uses self-induced congestion to locate the } \\
\text { range of } A_{P}\end{array}$ & 2002 \\
\hline & Pathload & $\begin{array}{l}\text { Jain and Dovrolis, } \\
\text { Georgia Tech. }\end{array}$ & UDP & $\begin{array}{l}\text { Self-Loading periodic streams, heuristic } \\
\text { inference }\end{array}$ & 2001 \\
\hline & Spruce & Strauss, et al., MIT & UDP & $\begin{array}{l}\text { Probe gap model, single bottleneck, and } \\
\text { pre-known } C_{P}\end{array}$ & 2004 \\
\hline & TOPP & $\begin{array}{c}\text { Melander, et al., } \\
\text { Uppsala Univ., Sweden }\end{array}$ & UDP & Trains of packet pairs, segmented regression & 2005 \\
\hline
\end{tabular}

* All systems implicitly use IP and lower layer protocols.

\section{3 工作原理及假设}

研究发现,上述 13 个系统都采用了探测-估算,再探测-再估算这样一种不断求精的主动度量方法,并且都基 于本质相同的工作原理. 简单地讲, 即 $R_{0}$ 以传输速率 $x$ 向 $R_{n}$ 发送一组探测数据包, 如果 $x>A_{P}$, 那么必定至少存在 一个链路, 它会以低于 $x$ 的速率传输这组数据包; 相反地, 如果 $x \leq A_{P}$, 那么这组数据包到达 $R_{n}$ 时的传输速率仍然 是 $x$. 通过发送不同速率的探测数据包, 并监测数据包传输速率的变化, 度量系统就能推断出探测速率与可用带 宽的关系,进而估算出可用带宽(或其所属范围).下面展开来讨论本工作原理.

设有 $m$ 个大小为 $s$ 的探测数据包, 被 $R_{0}$ 以速率 $x$ 发往 $R_{n}$. 相邻两个数据包被 $R_{i}$ 发送入 $L_{i}$ 的平均时间间隔(也 即相邻两数据包在 $L_{i}$ 上传输的平均时间间隔)为 $p_{i}, 0 \leq i \leq n-1$. 于是, 这组数据包在链路 $L_{i}$ 上的传输速率 $\mu_{i}$ 为

$$
\mu_{i}=\frac{s}{p_{i}}
$$

易知 $x=\mu_{0}$. 当这组探测数据包通过 $R_{i}$ 从链路 $L_{i-1}$ 传送到 $L_{i}$ 时,将面临两种情况:

第 1 种情况: $A_{i}<\mu_{i-1}(1 \leq i \leq n-1)$. 因为 $A_{i}<\mu_{i-1}$ 即 $C_{i}-\lambda_{i}<\mu_{i-1}$, 于是 $C_{i}<\mu_{i-1}+\lambda_{i}$. 通过 $R_{i}$ 进入 $L_{i}$ 的所有数据包的速 率之和已经超过了 $L_{i}$ 的带宽容量 $C_{i}$, 所以, $R_{i}$ 无法将所有进来的数据包(背景数据包加上探测数据包)都以它们 进入 $R_{i}$ 时的速率发送到 $L_{i}$ 中.在这种情况下, $R_{i}$ 将缓存部分数据包等待稍后再发送.这意味着在 $p_{i-1}$ 时间内, $R_{i}$ 
将不能发送 1 个以上的探测数据包, 于是, 相邻两探测数据包的间隔被 $R_{i}$ (或 $L_{i}$ ) 拉大了, 即 $p_{i}>p_{i-1}$. 根据公式(7), 因 为数据包大小 $s$ 不变, 于是 $\mu_{i}<\mu_{i-1}$.

在一些简化的网络模型中 ${ }^{[5]} p_{i}$ 和 $p_{i-1}$ 的关系还可以进一步量化为

$$
p_{i}=\frac{\mu_{i-1}+\lambda_{i}}{C_{i}} \cdot p_{i-1}
$$

但是在实际的网络环境中,公式(8)只能近似(而无法准确)地描述探测数据包间隔的变化 ${ }^{[18]}$. 由于本文主要 关注度量系统的基本问题, 考虑的是对上述 13 个系统都适用的分析, 所以在下面的讨论中, 仅认为当 $A_{i}<\mu_{i-1}$ 时, 有 $p_{i}>p_{i-1}$.

第 2 种情况: $A_{i} \geq \mu_{i-1}(1 \leq i \leq n-1)$. 因为 $A_{i} \geq \mu_{i-1}$ 即 $C_{i}-\lambda_{i} \geq \mu_{i-1}$, 于是 $C_{i} \geq \mu_{i-1}+\lambda_{i}$. 这说明 $L_{i}$ 有能力在不减速的情况下 传输所有数据包, 于是, 相邻两个探测数据包的间隔保持不变 $\left(p_{i}=p_{i-1}\right)$, 且探测数据包的传输速率也不变 $\left(\mu_{i}=\mu_{i-1}\right)$.

现以图 2 为例说明上述两种情况. 图中所有背景数据流都来自 $R_{C}$, 并流向 $R_{3}$. 源主机 $R_{0}$ 以 $\mu_{0}=3 \mathrm{Mbps}$ 的速率 向目标主机 $R_{3}$ 发送一组大小相同的探测数据包. 因为 $A_{1}<\mu_{0}$ 属于上述分析的第 1 种情况, 即探测数据包在 $L_{0}$ 上 的传输速率高于 $L_{1}$ 的可用带宽,所以,数据包间隔被 $R_{1}$ 扩大了 $\left(p_{1}>p_{0}\right)$. 接下来的 $A_{2} \geq \mu_{1}$ 属于第 2 种情况,所以, $R_{2}$

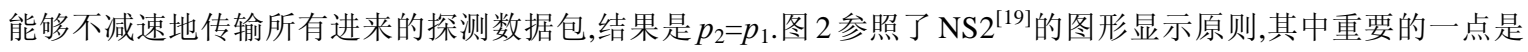
数据包的显示宽度与所在链路的带宽容量成反比.

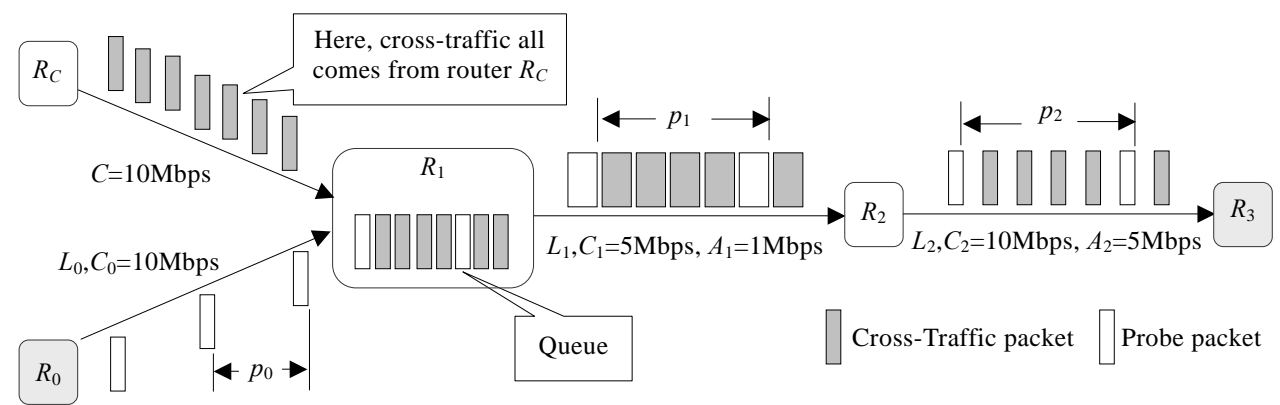

Fig.2 A typical probing process

图 2 一次典型的探测过程

在基于上述工作原理的同时,13 个度量系统还都(显式或隐式地)假设下面 4 点成立:

(1) 所有中间节点都采用先进先出(first-in-first-out,简称 FIFO)的缓冲队列管理算法;

(2) 背景数据流满足弗洛伊德(Fluid)模型,即数据包能够被拆分放入无限小的间隙中 ${ }^{[14]}$;

(3) 背景数据流的传输速率基本不变(或变化缓慢, 以至于在一次度量周期内可以被认为是恒定的);

(4) 源主机 $\left(R_{0}\right)$ 能够以高于可用带宽的速率发送探测数据包.

\section{4 相关工作}

Strauss 等人的研究表明,度量系统输出的结果总是存在难以消除的不确定性,这正是导致度量系统无法被 广泛应用和推广的首要原因 ${ }^{[14]}$.Jin 等人首次证明了主机的处理能力是影响度量结果准确性的一个重要因 素 ${ }^{[20]}$. 随后,Jain 等人进一步细化了度量可用带宽的场景, 并列举出度量过程中常见的 10 个概念误区 ${ }^{[21]}$. Lakshminarayanan 等人以实验数据说明,现有的度量系统大多只适用于传统网络环境 ${ }^{[22]}$, 当系统被用于 802.11 无线网络环境(或有线与无线并存的混合网络环境)时, 度量结果误差很大且无明显规律. 这些工作为设计和开 发准确、高效的度量系统提供了新的启示. 不足的是, 他们都只关注于度量过程的某些方面或仅针对一些具体 的应用场景,而未能全面地考虑度量系统中普遍存在的基本问题.本文基于文献[23]的工作,从度量系统的工作 原理、探测数据包在正反向路径上的传输过程、背景数据流和动态路由对度量过程的影响、各类实验平台上 的大规模实验及相关数据分析等多个方面扩展了内容,更加深入地研究了度量系统的基本问题. 


\section{2 单、双终端系统共有的基本问题}

下面,我们将度量系统中存在的基本问题分为 3 类:单、双终端系统共有的问题,单终端系统独有的问题以 及双终端系统独有的问题. 本节讨论单、双终端系统中都存在的 5 个基本问题:计时误差、低接入带宽、数据 包异常、路由器队列管理、结果验证.

\section{1 计时误差}

度量结果的准确性在很大程度上取决于系统计时的精确度.几乎每一个度量系统都要求精确地控制数据 包的发送时间,并准确获知数据包的接收时间. 虽然单、双终端系统的计时过程稍有区别, 但都存在计时误差的 问题. 在单终端系统中, 数据包发送时间和接收时间的记录与维护全部由 $R_{0}$ 负责, 并且 $R_{0}$ 所接收的数据包不是 探测数据包, 而是待测路径的中间节点 $R_{1}, \ldots, R_{n-1}$ 或目标主机 $R_{n}$ 发送给 $R_{0}$ 的应答数据包(reply packet). 双终端系 统由 $R_{0}$ 控制每个探测数据包在何时发送, 并由 $R_{n}$ 记录探测数据包于何时接收. 度量系统所有的后续计算(包括 对可用带宽的评估),都基于系统在每次度量周期中维护的发送-接收时间集合 $T_{S}$ 和 $T_{R}$.

$$
\begin{aligned}
& T_{S}=\left\{s_{1}, s_{2}, \ldots, s_{p}\right\}, s_{i} \text { 表示第 } i \text { 个指定数据包被发送的时间. } \\
& T_{R}=\left\{r_{1}, r_{2}, \ldots, r_{q}\right\}, r_{i} \text { 表示第 } i \text { 个指定数据包被接收的时间. }
\end{aligned}
$$

其中, $p$ 与 $q$ 分别为一次探测中需要记录时间的数据包发送事件和数据包接收事件的数量. 计时过程如图 3 所示, 在单终端系统中,集合 $T_{S}$ 和 $T_{R}$ 都由 $R_{0}$ 维护; 在双终端系统中,集合 $T_{S}$ 和 $T_{R}$ 分别由 $R_{0}$ 与 $R_{n}$ 维护.

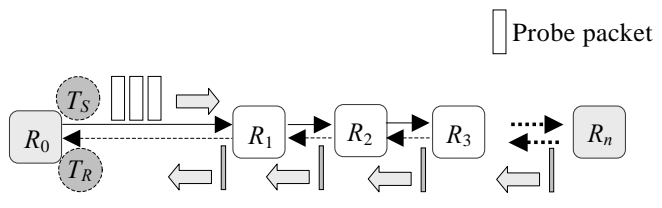

(a) Single end-host system

(a) 单终端系统

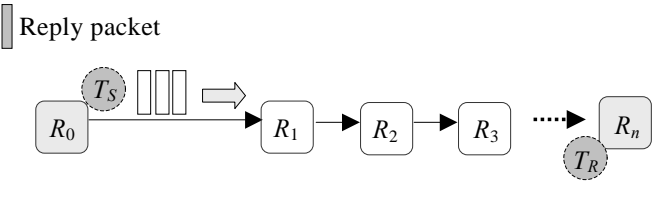

(b) Double end-host system

(b) 双终端系统

Fig.3 The send-receive timing processes of a measurement system

图 3 度量系统的发送-接收计时过程

为了说明计时误差,设 $t$ 是终端主机将数据包中的所有字节通过网卡放入物理传输媒介的时刻(或者是主 机将数据包中的所有字节通过网卡从物理媒介上完整接收进来的时刻), 并设 $t_{e}$ 是系统所记录的它完成这一操 作的时刻,则计时误差 $\varepsilon$ 可以表示为

$$
\varepsilon=\left|t-t_{e}\right|
$$

计时误差的存在,意味着系统所记录的数据包发送(或接收)时刻其实并不是该数据包实际被发送(或接收) 的时刻.计时误差 $\varepsilon$ 越大,度量结果就越不准确.

计时误差不仅在每个度量系统中都存在, 而且非常难以预测.数据包发送不及时是造成计时误差的一大原 因.通常认为,让 $R_{0}$ 在某个指定时刻发送出数据包,最好的方法是将度量系统的发送线程一直睡眠到那个时刻. 可问题是, 目前的主流操作系统(如 Windows 和 Linux) 都只能提供毫秒级的计时器(timer) ${ }^{[24]}$, 这对于需要精确计

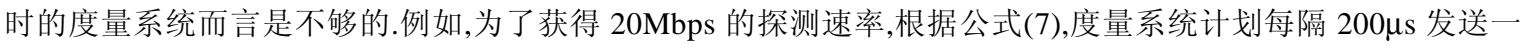
个 500 字节的 IP 包,为此, 它在相邻发送操作之间调用睡眠函数 sleep 将发送线程休眠 $200 \mu \mathrm{s}$. 为简化分析, 假设 发送操作所耗的时间可以忽略不计,且该度量系统在每次探测中仅发送一组共两个 IP 包.系统在某次探测中的 实际发包过程如图 4 所示. 由于计时器只能提供 $1 \mathrm{~ms}$ 的精度, 在某次实验中,操作系统让发送线程实际睡眠了 $700 \mu \mathrm{s}$,这导致 $\varepsilon=500 \mu \mathrm{s}$ 并且真正的探测速率仅有 5.7Mbps,这与期望的 20Mbps 相差相当大.

除了调用睡眠函数以外, 在指定时刻准时发送数据包的另一种方法是使用不间断循环(non-blocking loop $)^{[14]}$, 具体做法是, 让发送线程一直保持活动状态(不断地检测当前时间,并执行一些无意义的计算指令), 直到 发送出数据包才释放 CPU(如图 5(b)所示的示例代码). 与睡眠线程的方法相比, 不间断循环确实能够更及时地 
发送出数据包,但它也存在两大不足:一是发送线程强行占用了大部分 CPU 时间,因而会影响系统中其他功能的 执行. 这一点在单终端系统中尤其明显, 因为数据包的发送和接收都由 $R_{0}$ 来完成, 所以,采用不间断循环的数据 包发送方式必然严重影响接收线程的计时精度,进而导致 $T_{R}$ 严重失真.二是不间断循环并不能 $100 \%$ 地按时发 送数据包,它可能会被难以预测的系统事件(比如线程切换)打断.

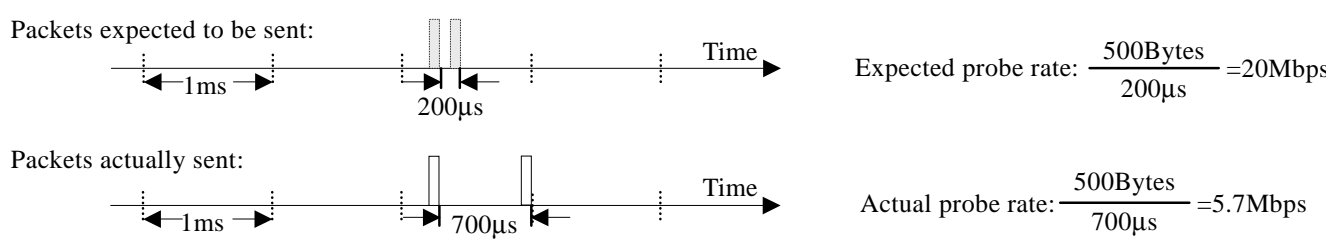

Fig.4 Timing error leads to unexpected probe rate

图 4 计时误差导致难以预测的探测速率

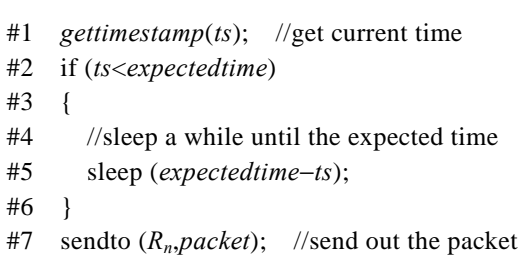

(a) Use sleep function to send a packet at specific time

(a) 使用 Sleep 方法在指定时刻发送数据包

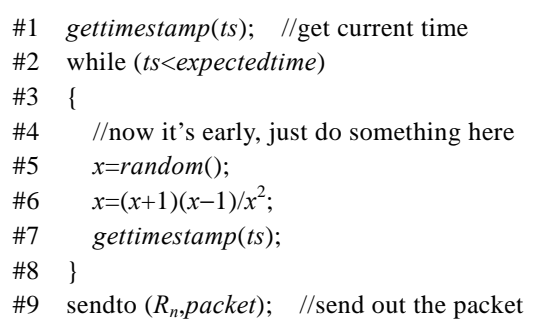

(b) Use non-blocking loop to send a packet at specific time (b) 使用非中断循环在指定时刻发送数据包

Fig.5 The example code of two packet-sending methods

图 5 两种数据包发送方式的示例代码

线程切换也是造成计时误差的一大原因.操作系统通过多线程技术来支持并发任务的执行,它不断地切换 线程, 从而确保每个线程都能获得一定的执行时间. 度量系统的线程在运行过程中也不可避免地会被线程切换 打断; 当它被打断时,核心资源(如 CPU)将被其他线程占用.线程切换带来的可能后果是:该发送数据包的时候不 能发送,或者该接收数据包的时候不去接收. 在这种情况下, 时间集合 $T_{S}$ 和 $T_{R}$ 就是断裂的 ${ }^{[18]}$, 即它们包含了不必 要的线程切换开销.尽管可以在度量结束之后,根据操作系统的指令审计来检测相邻指令间是否存在异常间隔, 从而判断是否发生了线程切换.但即使如此,目前仍没有可行的办法能够恢复已经断裂的 $T_{S}$ 和 $T_{R}{ }^{[24]}$.

网卡采用的中断技术是一个容易被忽略的计时误差来源.目前,不少高速网卡(比如 SysKonnect 9843)都采 用了中断合并技术(interrupt coalescence ${ }^{[25]}$. 传统网卡每接收到一个数据包就会产生一个中断信号, 让系统读取 它缓存中的数据.采用了中断合并技术后,当网卡接收到一个数据包时,它生成一个中断信号但并不立即发送该 信号,相反, 它会推迟片刻才发送这个信号. 这样做的目的是,期望在这片刻中,网卡能够接收到额外的数据包,而 这些额外的数据包将不会产生新的中断. 因此在有限的时间内,多个数据包可以共用一个中断信号, 从而节省了 网卡的资源开销. 文献[20,25]中的实验证明了中断合并技术确实能够有效提高网卡的吞吐量. 不过, 也正是因为 该项技术的存在,使得操作系统无法保证它能获知所有数据包被接收的真实时间.比如,两个数据包共用了同一 个中断信号, 那么,操作系统能够获知的只是前一个数据包被接收的时间(它触发了中断信号), 而无法得知后一 个数据包是何时被接收的. 这个问题可以通过修改网卡的驱动程序从而读取网卡寄存器(NIC register)来解决. 但是,网卡的驱动随着生产厂商及主要处理芯片的不同而有很大差别, 因此, 在一台主机中的修改无法有效地应 用到其他主机上.另外,修改驱动程序需要极高的系统权限,存在很大的安全风险,所以这个方案并不可行.

系统调用延迟也是计时误差的重要来源. 系统调用延迟是指从调用操作系统函数到获得该函数返回值(包 括 null)的这段时间. 度量系统往往忽略了执行系统调用所需要的时间, 或简单地假设系统调用延迟为 0 , 即函数 
被调用的时刻也就是该函数执行完毕的时刻.但事实上,系统调用明显是耗费时间的,不同的系统调用以及同一 系统调用在不同软、硬件平台上(或不同负载情况下)的时间开销也会不同.表 2 列出了度量系统中常用的 3 个 系统调用: 获取系统当前时间(get timestamp)、读网络套接字(read socket)和写网络套接字(write socket). 后两个 系统调用是接收和发送数据包的核心操作.表 2 给出了系统调用的最小 (min)、平均(ave)和最大(max)延迟. 最小 和最大延迟分别是指连续 50 个系统调用的延迟中的最小值和最大值. 这里重点介绍平均延迟的测量方法. 现连 续调用共 51 次 gettimestamp 函数(事后确认其间没有发生线程切换),假设第 $i$ 次调用该函数后获得的时间标签 为 $t_{i}$, 那么, gettimestamp 的平均延迟 $d_{T}$ 为

$$
d_{T}=\left(t_{51}-t_{1}\right) / 50
$$

Table 2 The delay of system call

表 2 系统调用延迟

\begin{tabular}{c|c|ccc|ccc|ccc}
\hline \multirow{2}{*}{ Operating system $^{*}$} & \multirow{2}{*}{ CPU } & \multicolumn{3}{|c|}{ Get timestamp $(\mu \mathrm{s})$} & \multicolumn{3}{c|}{ Read socket $(\mu \mathrm{s})$} & \multicolumn{3}{c}{ Write socket $(\mu \mathrm{s})$} \\
\cline { 3 - 9 } & & min. & ave. & max. & min. & ave. & max. & min. & ave. & max. \\
\hline Linux 2.4.1 & Intel Xeon 2.4 GHz & 0.8 & 0.9 & 1.0 & 28.7 & 31.4 & 33.3 & 27.6 & 30.7 & 32.2 \\
Linux 2.4.1 & Intel P4 2.0 GHz & 0.8 & 1.0 & 1.2 & 29.5 & 32.9 & 34.6 & 28.9 & 32.6 & 34.8 \\
Linux 2.4.1 & AMD AthlonXP 2500+ & 0.7 & 0.8 & 0.9 & 28.9 & 31.1 & 33.4 & 29.2 & 31.6 & 33.5 \\
FreeBSD 4.8 & Intel P3 700 MHz & 4.3 & 4.6 & 5.0 & 45.2 & 48.9 & 50.5 & 45.1 & 47.0 & 49.5 \\
Solaris 2.8 & Sparc 400 MHz & 0.5 & 0.5 & 0.5 & 33.6 & 36.8 & 37.9 & 32.3 & 35.2 & 38.1 \\
Mac OS X 10.2 & Power G4 1.0 GHz & 1.8 & 1.9 & 2.0 & 43.2 & 45.8 & 48.9 & 40.2 & 43.5 & 45.3 \\
Windows XP SP2 & Intel P4 2.8 GHz & 0.9 & 1.0 & 1.1 & 38.8 & 40.1 & 42.4 & 35.4 & 38.6 & 40.7 \\
\hline
\end{tabular}

* For the first six OSs, we invoke gettimeofday() to get timestamp; for Windows, we invoke QueryPerformanceCounter().

We invoke socket functions $\operatorname{recv}()$ and $\operatorname{send}($ ) for read and write socket system calls, respectively.

获取其他系统调用延迟的方法与获取 $d_{T}$ 稍有不同. 在度量 write socket 的平均延迟 $d_{W}$ 时, 我们连续不间断 地执行 50 次 write socket 操作,每次操作都向 socket 中写入 500 字节的内容.紧接着在第 1 次操作前以及第 50 次操作后,我们调用了 gettimestamp 函数,得到的时间标签分别为 $t_{1}$ 和 $t_{2}$.于是有

$$
d_{W}=\left(t_{2}-t_{1}-d_{T}\right) / 50
$$

计算 read socket 平均延迟的方法与计算 $d_{W}$ 的方法相同.需要说明的是,由于事先分配了足够大的缓存空间, 并且预写入了足够多的数据, 所以每次调用 read socket 操作时, socket 缓存中总是有至少 500 字节的数据可读. 总的来讲,为了降低计时误差, 我们需要整合并优化从度量软件、操作系统到底层硬件等所有涉及到的软、硬 件资源.比如,Cprobe 采用 SGI 公司的 IRIX 操作系统和硬件体系并做大量优化后,宣称能达到 40ns 的时间粒度. 但从我们的实验看来,要想在通用操作系统如 Windows 和 Linux 上开发出具有同等计时粒度的度量软件还非常 困难.

\section{2 低接入带宽}

回顾第 1.3 节,度量系统正常运行的前提之一是它能够以高于可用带宽的速率发送探测数据包.然而,过低 的接入带宽 $\left(C_{0}\right)$ 将严重限制度量系统评估网络路径整体带宽情况的能力. 例如, 对一个通过 56Kbps 调制解调器 接入互联网的主机而言, 度量系统输出的结果始终不会高于 56Kbps(如图 6 所示). 这并不是度量系统出错了, 相 反, 它是正确的.

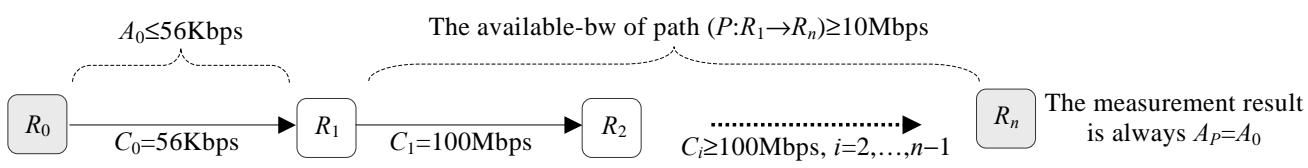

Fig.6 $L_{0}$ decides end-to-end available bandwidth when $C_{0}$ is comparatively low

图 6 接入带宽 $C_{0}$ 过低时,可用带宽往往由 $L_{0}$ 决定

根据公式(6),端到端可用带宽正是待测路径中最小的链路可用带宽.但问题是在低接入带宽的情况下,度量 到的其实总是 $A_{0}$. 这样一种度量结果往往不是用户想要的,此时, 用户更希望了解其他部分网络路径的可用带 
宽.低接入带宽问题是带宽度量研究中的一个富有挑战性的问题,尽管有些基于概率的方法(如 Monte Carlo ${ }^{[4]}$ ) 能够部分地解决这个问题,但至今没有相关的软件系统出现.

现在,大部分主机都能以 $10 \mathrm{Mbps}, 100 \mathrm{Mbps}$, 甚至 $1000 \mathrm{Mbps}$ 的速率访问互联网,这意味着在很多情况下, $C_{0}$ 并不像图 6 中的 $56 \mathrm{Kbps}$ 这么低.但是,源主机 $R_{0}$ 作为待测路径中必须安装度量系统的终端节点,一般都处于网 络分层体系的底部,它的接入带宽一般都低于上层 ISP 的带宽.从 2002 年起,大量 Tier-1 和 Tier-2 ISP 都开始基 于 OC-192(10Gbps)和 OC-768(40Gbps)光纤链路来组建骨干网络 ${ }^{[26]}$. 高速骨干网络带来的问题是,如果待测网络 路径的大部分中间链路都属于高速率骨干网路, 那么, 度量系统很可能会由于接入带宽过低而无法有效地评估 整个路径的带宽情况.

低接入带宽问题不仅取决于主机的网络配置,还取决于主机的数据包处理能力.在一个 $1000 \mathrm{Mbps}$ 的局域 网中, 我们简单地测试了 6 个不同的主机所能达到的最大传输速率. 在实验中, 每个系统连续不间断地发送出 3000 个 1500 Bytes 的探测数据包,那么,传输速率就等于 4.5MBytes 除以发送所需的时间,测试结果见表 3. 我们 发现:

第一,主机的最大传输速率总是低于其网卡(NIC)的处理能力(这也是很自然的事情);

第二,网卡的利用率随着链路带宽的增长呈明显的递减趋势:当网卡是 $10 \mathrm{Mbps}$ 时,其利用率高达 $86 \%$ $89 \%$; 然而, 当网卡为 $1000 \mathrm{Mbps}$ 时, 其利用率只有 $25 \%$ 34\%. 这意味着, 受限于通用计算机系统的数据包处理能力, 即 使在配置了高带宽连接的情况下,主机也难以获得较高的传输速率.

Table 3 The maximum data-rate achieved by end-host

表 3 主机能达到的最大速率

\begin{tabular}{cccccc}
\hline \multirow{2}{*}{ Operating system } & \multirow{2}{*}{ CPU } & \multicolumn{3}{c}{ Achievable data-rate (Mbps) } \\
\cline { 3 - 5 } & & $N I C=10^{*}$ & $N I C=54$ & $N I C=100$ & $N I C=1000$ \\
\hline Linux 2.4.1 & Intel P4 2.0 GHz & 8.8 & 38.3 & 77.2 & 323.0 \\
Linux 2.4.1 & AMD AthlonXP 2500+ & 8.9 & 39.1 & 78.3 & 340.2 \\
FreeBSD 4.8 & Intel P4 2.0 GHz & 8.8 & 33.6 & 72.5 & 299.2 \\
Solaris 2.8 & Sparc 400 MHz & 8.6 & 36.0 & 73.4 & 310.0 \\
Mac OS X 10.2 & Power G4 1.0 GHz & 8.6 & 36.5 & 68.6 & 256.7 \\
Windows XP SP2 & Intel P4 2.8 GHz & 8.8 & 36.3 & 70.6 & 280.2 \\
\hline
\end{tabular}

* "NIC $=x$ " means the NIC bandwidth is $x \mathrm{Mb} / \mathrm{s}$. The $10 \mathrm{Mbps}, 54 \mathrm{Mbps}, 100 \mathrm{Mbps}$, and $1000 \mathrm{Mbps}$ NICs are

TP-LINK TE-2029 PCI, Intel Pro/Wireless 3945ABG, Realtek 8139, and SysKonnect 9843, respectively.

\section{3 数据包异常}

度量系统通过监控探测数据包来感知网络路径的带宽状况, 因此很容易受到数据包异常 (pathology)的干 扰. 数据包异常是指端到端数据传输过程中发生的异常事件, 主要有包丢失(packet loss)、乱序传送(out-of-order delivery)、包重复(packet replication)和包破坏(packet corruption). 在很多情况下, 数据包异常会导致 $T_{R}$ 中包含不 合理的数据, 进而影响度量过程的准确性. 举例来说, 如果探测数据包在传输途中被某个路由器给丢弃了 (包丢 失), 度量系统就无法维持完整的时间集合 $T_{R}$; 同样, 如果数据包在途中被乱序传送,那么,根据 $T_{R}$ 所推算出来的数 据包间隔将出现难以理解的负值; 另外,当包重复出现时,假设有两个完全一样的探测包抵达 $R_{n}$, 系统将无法确 认需要向 $T_{R}$ 中放入哪一个探测包的接收时间(只能且必须放一个); 最后,如果一个探测数据包在途中被意外地 破坏了,那么,系统很可能由于无法识别它的特征信息而将它误认为是背景数据包,这同样会影响到系统对 $T_{R}$ 的 维护.

早在 1994 1995 年间,Paxson 就在分布于世界各地的 37 台主机上安装了 network probing daemon(NPD)程 序, 并在大规模网络实验的基础上统计了数据包异常现象发生的概率 ${ }^{[18]}$.2005 年 10 月,我们在 8 台主机联结而 成的覆盖型网络(overlay network)上再次运行了 NPD,分两次(第 1 次 5000 个,第 2 次 8000 个)共收集了约 13000 个 TCP 连接的记录(trace), 并用 Paxson 的方法 ${ }^{[18]}$ 统计了数据包异常的概率. 表 4 给出了这 8 台主机所在 机构的名称以及主机之间的跳转数(hop count), 跳转数由 traceroute ${ }^{[27]}$ 获得. 比如从 $I O S_{1}$ 到 $G U C A S_{2}$ 的 IP 包要经 过 11 个跳转,这说明从 $I O S_{1}$ 到 $G U C A S_{2}$ 的网络路径共包含 11 个链路.部分主机(如 Telecom)不生成 ICMP 包, 
所以到这些主机的路径无法用 traceroute 获知跳转数(用?标出).此外,两台主机之间往返路径的跳转数可能是不 一样的(用斜体标出).

Table 4 The hop count between any two nodes of the overlay experimental network 表 4 覆盖型实验网络中任意两节点间的 IP 跳转数

\begin{tabular}{ccccccccc}
\hline & $I O S_{1}$ & $I O S_{2}$ & $G U C A S_{1}$ & $G U C A S_{2}$ & Tsinghua & PKU & USTC & Telecom \\
\hline$I O S_{1}$ & - & 3 & 10 & 11 & 14 & 14 & 16 & $?$ \\
$I O S_{2}$ & 3 & - & 11 & 12 & 15 & 15 & 17 & $?$ \\
$G U C A S_{1}$ & 10 & 11 & - & 2 & 12 & 12 & 16 & $?$ \\
GUCAS & 12 & 12 & 2 & - & 13 & 13 & 16 & $?$ \\
Tsinghua & 14 & 15 & 12 & $?$ & - & 13 & 16 & $?$ \\
PKU & $?$ & $?$ & $?$ & $?$ & 13 & - & 16 & $?$ \\
USTC & 16 & 17 & 15 & 16 & 16 & $?$ & - & $?$ \\
Telecom & 15 & 17 & 14 & 15 & 14 & 15 & 14 & - \\
\hline
\end{tabular}

The meaning of symbols is as follows. -: no connection to itself; ?: hop count unknown; italic font: the forward \& reverse paths between a pair of nodes comprise different number of routers. Specifically, IOS, GUCAS, Tsinghua, PKU, USTC, Telecom are Institute of Software, Graduate University of the Chinese Academy of Sciences, Tsinghua University, Peking University, University of Science and Technology of China, and Beijing Telecom, respectively.

图 7 给出了在 Paxson 的两个 TCP 连接记录集合 $\left(N_{1}, N_{2}\right)$ 和我们的记录集合 $\left(N_{3}, N_{4}\right)$ 上计算出的包异常概率.

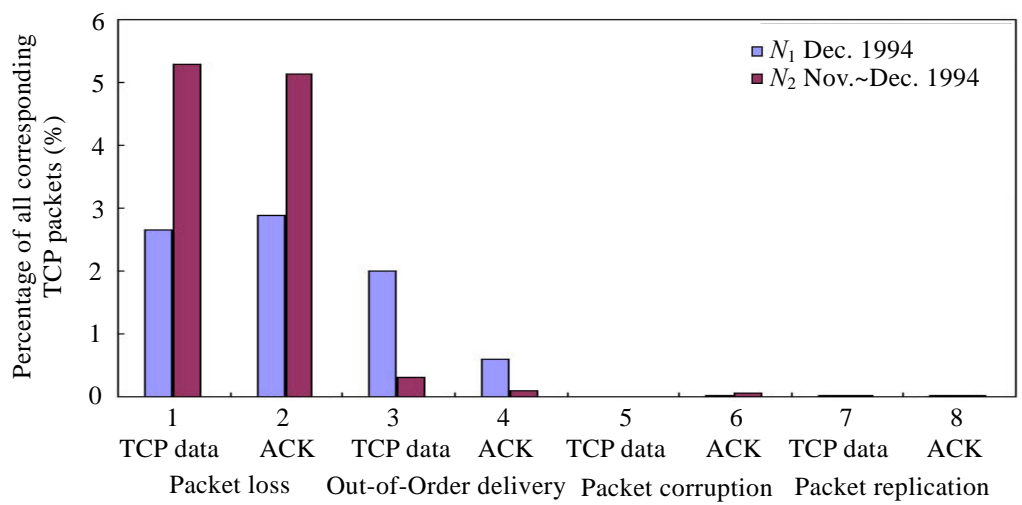

(a) Pathologies measured by Paxson in 1994 1995

(a) Paxson 在 1994 1995 年间度量到的数据包传输异常

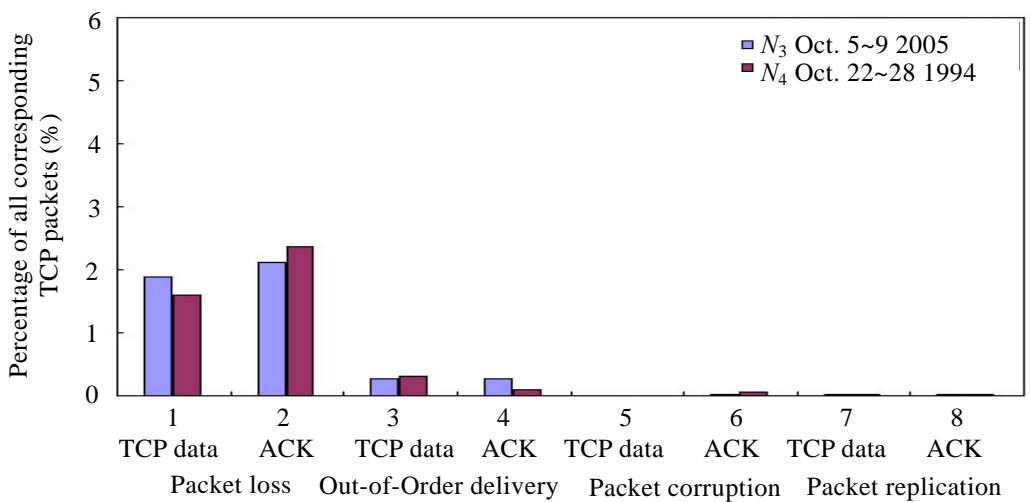

(b) Pathologies computed from our datasets in 2005

(b) 从 2005 年数据集中计算出来的数据包传输异常

Fig.7 The ratios of end-to-end packet pathologies

图 7 数据包异常事件发生的比率 
集合 $N_{1}, N_{2}, N_{3}$ 和 $N_{4}$ 分别是在 1994 年 12 月、1995 年 11 12 月、2005 年 10 月初和 2005 年 10 月末收集的. 需要注意的是,NPD 只能记录和分析 TCP 数据包(TCP data)与 TCP 应答包(ACK)的异常现象.但鉴于 TCP 是当 今网络中最主要的通信协议,所以,基于 NPD 程序的分析基本上能够反映网络中数据包异常的真实情况.如图 7 所示, 在所有的 TCP 数据包中的包丢失的概率分别为 $2.7 \%\left(N_{1}\right), 5.2 \%\left(N_{2}\right), 1.9 \%\left(N_{3}\right)$ 和 $1.7 \%\left(N_{4}\right)$. 不难看出, 在这 4 个集合中,上述 4 种包异常现象都是存在的,其中以包丢失和乱序传送最为突出.

很难从根本上完全消除数据包异常对可用带宽度量过程的影响. 根据第 1.3 节中介绍的工作原理,度量系 统需要不断发送大量的探测数据包, 并推断探测速率与可用带宽的关系, 从而估算可用带宽. 同时, 度量系统还 要求源主机的发送速率能够大于可用带宽, 这意味着度量系统的主动探测将导致局部的网络拥塞, 即至少 1 个 路由器的缓冲队列会增大. 当发生网络拥塞时, 上述 4 种数据包异常现象 (特别是包丢失)就有可能发生 ${ }^{[18]}$. 关键 的问题是, 如果度量系统所发送的探测数据包引发了数据包异常现象, 而这些现象又与待测路径本身就存在的 数据包异常现象重叠在一起, 那么, 从终端主机 $\left(R_{0}\right.$ 和 $\left.R_{n}\right)$ 的角度要想准确获知造成包异常的原因, 并正确判断探 测速率与可用带宽之间的关系是非常困难的.

\section{4 路由器队列管理}

尽管度量系统都(显式或隐式地)假设中间路由器采用 FIFO 缓冲队列管理算法,但事实却并非如此 ${ }^{[28]}$.例如, 已有相当多的路由器采用了主动队列管理(active queue management, 简称 AQM)算法 ${ }^{[29]}$. 与 FIFO 相比, AQM 的 设计思想是尽可能提前避免网络拥塞, 而不仅仅是顺序处理进入路由器的数据包. 当数据链路即将拥塞时(链路 的利用率高于某个预定的阈值,如 80\%),AQM 路由器会主动丢弃部分数据包以降低拥塞的可能性.

但是, 从带宽度量的角度来讲, AQM 将导致三大问题:第一,当探测数据包被丢弃时,网络拥塞可能只是将要 发生而不是一定发生, 所以当数据包被 $\mathrm{AQM}$ 路由器丢弃后, 就推断拥塞已经发生(或认为探测速率高于可用带 宽) 是不准确的; 第二, 终端主机 $R_{0}$ 和 $R_{n}$ 无法预知 $\mathrm{AQM}$ 会导致多大的丢包率, 也无法将 AQM 导致的丢包与节点 或链路出错导致的丢包区分开来; 第三, 当探测数据流给待测路径带来局部拥塞时,AQM 路由器可能会丢弃路 径中正常传输的数据流 (即背景数据流). 这违背了度量系统的设计初衷, 即度量是为了更好地传输数据, 而不是 影响或损害背景数据流的传输. 总之, 如果瓶颈链路 $L_{i}$ 的入口节点 $R_{i}$ 采用的是 AQM 而不是 FIFO, 那么, 度量系 统将难以通过观察探测数据包传输情况的方法来准确判断探测速率与可用带宽的关系.

然而,即使所有节点都采用 FIFO, 在某些情况下,网络拥塞也会导致数据包间隔的异常变化,从而掩盖探测 速率与可用带宽的真实关系. 比较典型的是,尽管探测速率小于可用带宽, 但是相邻数据包的间隔却被压缩了, 而不是像第 1.3 节中分析的那样保持不变. 如图 8 所示, $R_{0}$ 向 $R_{3}$ 发送两个探测数据包,这两个探测数据包在 3 个 背景数据包之后进入 $L_{1}, 5$ 个数据包都将被 $R_{2}$ 发送到 $L_{2}$ 上. 当第 1 个探测数据包进入 $R_{2}$ 时, 由于 $R_{2}$ 还没有将 3 个背景数据包都发送出去, 所以,这个探测数据包被缓存在 $R_{2}$ 中, 直到 3 个背景数据包都进入 $L_{2}$ 后才被发送. 当 第 2 个探测数据包进入 $R_{2}$ 时, 由于前面的数据包都已经被处理了, 因此, 该数据包被 $R_{2}$ 直接发送入 $L_{2}$. 最后的结 果是当 $A_{2} \geq \mu_{1}$ 时 $p_{2}<p_{1}$, 即数据包间隔被压缩了.

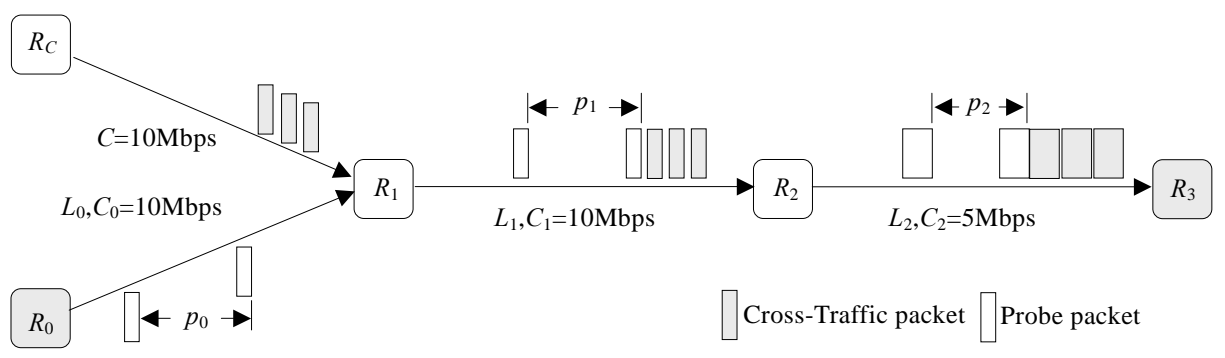

Fig.8 The compression of probe packet dispersion $\left(p_{0}=p_{1}>p_{2}\right)$

图 8 被压缩的数据包间隔 $\left(p_{0}=p_{1}>p_{2}\right)$ 
无论如何,只要探测数据包的间隔变化无法正确反映出探测速率与可用带宽的关系,就会影响度量系统的 带宽估算. 异常的间隔变化一般都是由突发的背景数据流引起的 ${ }^{[18]}$. 鉴于此,很多度量系统倾向于采用长数据包 队列(即一组几十个或上百个数据包)来探测网络, 并综合考虑队列内数据包间隔的平均变化, 期望通过这种方 法弱化突发背景数据流的影响. 但数据包队列的长度选择却是一个大问题, 队列过长会干扰路径中正常的数据 传输, 且度量过程耗时太长; 队列过短又无法准确捕捉到探测速率与可用带宽的关系. 另外, 互联网的高度动态 性和不确定性使探测数据包队列的合理配置变得更加困难. 比如,一个在某些路径中工作得很好的数据包队列 对其他路径却未必合适 ${ }^{[21]}$. 即使在同一个待测路径中,最佳的队列配置也不是固定的,它将随着路径中各节点的 属性和负载状况而变化. 目前, 尚未有可行的办法能够从根本上消除异常的间隔变化给度量系统带来的负面 影响.

\section{5 结果验证}

每个度量系统都必须确保它所输出的度量结果与真实值是一致的,然而, 度量结果的验证过程却比较复杂. 根据公式(6),端到端可用带宽是指整个待测网络路径的可用带宽, 它由该路径的最小链路可用带宽决定.关键的 问题在于,要知道哪段链路的可用带宽最小,就必须获知待测路径中每一段链路的可用带宽.

常见的验证方法有 4 种:

第 1 种验证方法是将批量 TCP 吞吐量(bulk TCP throughput,简称 BTT)等同于可用带宽.使用这种方法的人 认为,可用带宽就等于 $R_{0}$ 到 $R_{n}$ 所能达到的 BTT, 并常常在度量之前获取 BTT 的值以便与度量到的可用带宽进 行比较. 但事实上, 可用带宽与 BTT 是不同的概念, 前者是指网络路径的端到端空闲带宽容量, 这个容量是独立 于传输协议的; 而后者是指 TCP 连接所能够达到的最大传输速率, 它与 TCP 协议本身的多种机制(如流量控制 和拥塞避免)紧密相关.所以本验证方法是不正确的.

下面通过实验来证明这一点. 实验平台是一个由 7 台高性能 PC 顺序连接而成的线性网络(如图 9 所示).节 点 $R_{3}$ 和 $R_{4}$ 上运行 Iperf ${ }^{[30]}$, 以产生从 $R_{3}$ 到 $R_{4}$ 的单链路恒定(one-hop-persistent, 简称 OHP)TCP 数据流,这样, $L_{3}$ 就是瓶颈链路且 $A_{P}=A_{3}$. 通过调节 Iperf 的参数来控制从 $R_{3}$ 到 $R_{4}$ 的数据流速率, 我们就能在开始度量之前设置好 $A_{P}$, 并真实、可靠地比较 BTT 与 $A_{P}$ 的差别.此外, 获取 BTT 的方法大体上有两种:一种是扩大 $R_{0}$ 与 $R_{6}$ 的 TCP 缓 存窗口(window); 另一种是在 $R_{0}$ 与 $R_{6}$ 之间采用多个并发的 TCP 连接来传输大量数据. 在本文的多处实验中仅使 用 OHP 数据流, 一是因为它是最简单的背景数据流, 其他类型的数据流可以由它组合而成 ${ }^{[1]}$; 二是因为它已经足 够用来验证我们的观点.

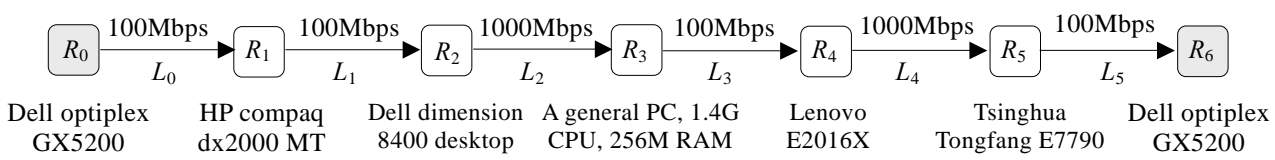

Fig.9 In-House experiment platform in the Institute of Software (IOS), the Chinese Academy of Sciences

图 9 在中国科学院软件研究所(IOS)内部搭建的实验平台

实验结果如图 10 所示. 我们发现:首先, 单个 TCP 连接所能达到的吞吐量总是小于 $A_{P}$; 其次, BTT 不是一个 固定的数值(这一点与可用带宽一样), 不同方法获得的 BTT 也是不一样的; 再次, 扩大 TCP 两端的缓存窗口 (window) 确实能够获得更大的 BTT, 但此时,BTT 也还是低于可用带宽; 最后, 当使用并发 TCP 连接时, 能够获得 相当高的 BTT(部分甚至高于 $A_{P}$ ). 这说明, 当 $A_{P}$ 较低时, 多个并发的 TCP 连接会与路径中的背景数据流争夺带 宽,严重影响背景数据流的传输. 总之, 将 BTT 或者某种传输协议所达到的最大吞吐量等同于可用带宽的验证方 法是不正确的. 


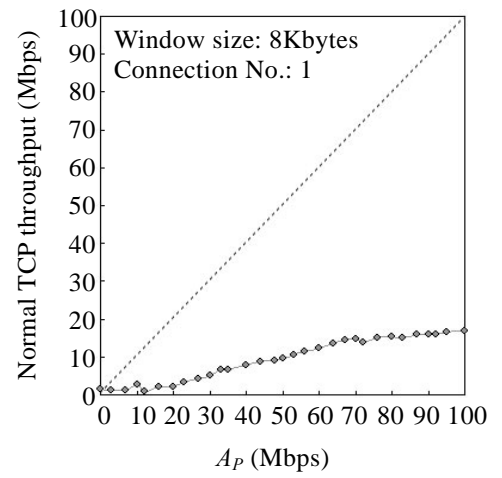

(a) Available-BW vs. normal TCP throughput

(a) 可用带宽与一般 TCP 吞吐量的对比

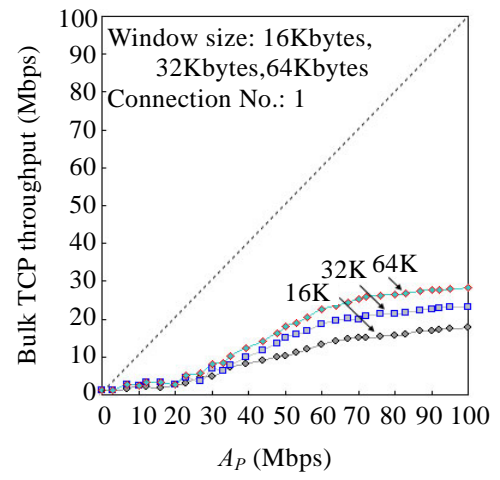

(b) Available-BW vs. BBT (large window size)

(b) 可用带宽与 BBT 的对比 (扩大 TCP 窗口)

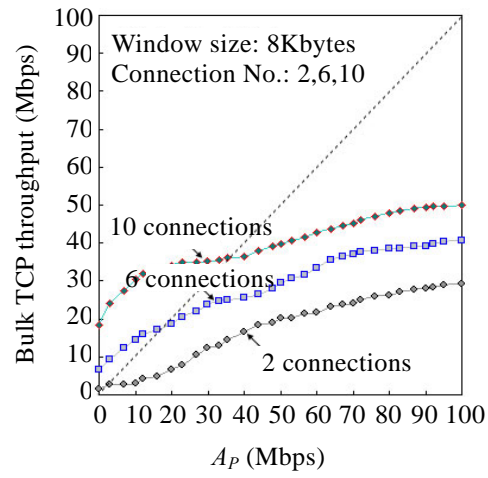

(c) Available-BW vs. BBT (multiple connections)

(c) 可用带宽与 BBT 的对比 (增加连接数量)

Fig.10 Comparison of available-bandwidth and BTT

图 10 可用带宽与 BTT 的比较

第 2 种验证方法是在中间节点上运行统计软件(如 $\mathrm{MRTG}^{[31]}$ )来计算可用带宽.这种方法能够收集到待测路 径上各节点的流量统计信息,并从这些信息中推算出可用带宽.与第 1 种方法相比,本方法基于路由器的统计信 息,因此更加真实、可靠.但是,本方法要求拥有在所有中间节点上安装和运行软件的权限,而这恰恰是问题之所 在, 因为这样的权限在任何一个网络中都是非常宝贵的, 它仅掌握在包括网络管理员在内的少数人手中.此外, 一条网络路径往往会跨多个 ISP 的管辖范围,比如,从中国科学院软件研究所到清华大学的一条网络路径就包 括 13 个链路,涵盖了 5 个 ISP 的管辖范围(如图 11 所示). 这种跨 ISP 的多跳转路径, 随着近 10 年互联网的爆炸 式膨胀已经变得非常普遍.要想同时在多个 ISP 中的路由器上拥有安装和运行软件的权限是相当困难的,所以, 本方法仅适用于类似校园网的单个 ISP 内部的网络路径,而不适用于跨 ISP 的网络路径.

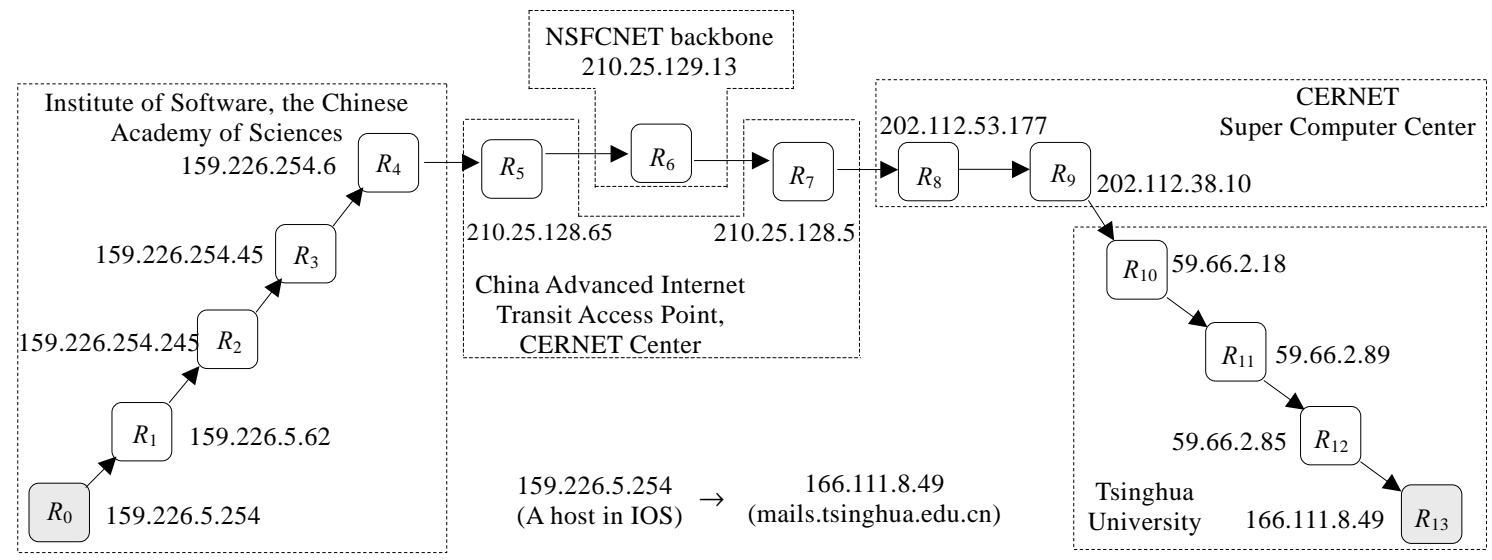

Fig.11 ISPs crossed by a network path

图 11 一条网络路径穿过的多个 ISP

第 3 种验证方法是使用网络模拟器(如 $\mathrm{NS}^{[19]}$ ), 这也是大多数度量系统首选的验证方法.网络模拟器能够根 据需要动态配置多种多样的网络环境,且能够记录模拟环境中几乎所有事件的信息.根据我们在 NS2 上近 5 年 的开发和使用经验,我们认为模拟器能够非常有效地验证理论算法的合理性,但是它无法确保实现该算法的软 件系统能够在真实网络环境下有效运行. 相关研究也说明,网络模拟器毕竟是建立在对真实网络的抽象(或简 
化)模型之上的,它所模拟出来的网络环境只能够近似一一而永远不可能等于一一真正的网络 ${ }^{[32]}$.

最后,还可以在机构内部的实验网络(in-house experimental network)中检验度量系统.对于可用带宽的度量 而言, 实验网络采用线性结构即可 (如图 9 所示), 节点可以是 PC 或路由器.节点上需要安装软件以设置链路的网 络属性, 从而模拟特定的场景. 较为知名的软件有流量生成软件 $\operatorname{Iperf}^{[30]}$ 和网络属性设置软件 NIST Net ${ }^{[33]}$ 等. 在 实验网络中检验度量系统, 能够获得比网络模拟器更真实的信息, 而且更有利于全面地测试度量系统. 这种方法 存在的问题是:基于实验网络的验证结果常常难以令人信服地被应用到广域网中.例如,IGI 系统在一个特定的 实验网络中给出了让人满意的度量结果 ${ }^{[6]}$, 但却在用于度量 400 条普通网络路径时表现糟糕 ${ }^{[14]}$. 事实上, 互联网 是如此庞大、复杂和多变,它有太多的特征是网络模拟器和实验网络环境所不能模拟的.

\section{3 单终端系统独有的基本问题}

上述 5 大类基本问题是单、双终端系统中都存在的.接下来研究单终端系统独有的基本问题.

单终端系统的探测度量机制建立在各种 echo 技术上,简单来讲,即 $R_{0}$ 向待测路径中发送特殊的探测包,中 间节点和目标主机在接收到这些探测包后会反馈给 $R_{0}$ 一些应答数据包.单终端系统的分析和估算工作主要就 建立在 $R_{0}$ 对探测数据包的发送和对应答数据包的接收上.Echo 技术有多方面的优点(比如仅需在 $R_{0}$ 上安装软 件), 然而它也给单终端系统带来了很多问题,这些问题主要集中在反向路径(从 $R_{n}$ 到 $R_{0}$ )中各方面因素对应答数 据包的影响上.

\section{1 背景数据流}

背景数据流是反向路径中影响度量过程的首要因素,下面以 BNeck 系统为例来说明这一点.BNeck 采用的 echo 探测技术充分利用了 ICMP 协议的特点. 在每次探测过程中,BNeck 以速率 $x$ 发射一组共 $2 N$ 个大小为 $s$ 的 IP 包 $(N<256)$. 这组 IP 包的 time-to-live(TTL)域值被 BNeck 有目的地设置成线性递增和递减的对称数对(如 图 12(a)所示), 这样的设置有如下特殊作用: 当这组 IP 包经过 $R_{1}$ 进入 $L_{1}$ 时, 首、尾两个数据包由于 TTL 值都是 1 , 就会被 $R_{1}$ 丢弃, 同时, $R_{1}$ 生成两个对应的 ICMP TE 包,并将其返回给 $R_{0}$, 其余的 2(N-1) 个 IP 包的 TTL 值被 $R_{1}$ 分别减 1 后发送到 $L_{1}$ 上. 以此类推(如图 12(b)所示), 当这组数据包从 $L_{i-1}$ 进入 $R_{i}$ 时, 首、尾两个 IP 包的 TTL 值 仍然是 1 , 因此, 它们会被 $R_{i}$ 丢弃. 同时, $R_{i}$ 发送两个 ICMP TE 包给 $R_{0}$; 如果 $R_{i}$ 不是 $R_{n}$, 则剩余的所有 IP 包在 TTL 值减 1 后被传送到 $L_{i}$ 上.

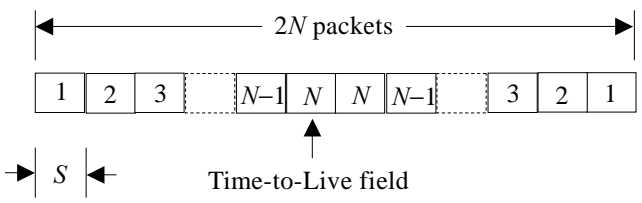

(a) Packet train configuration

(a) 数据包队列的配置

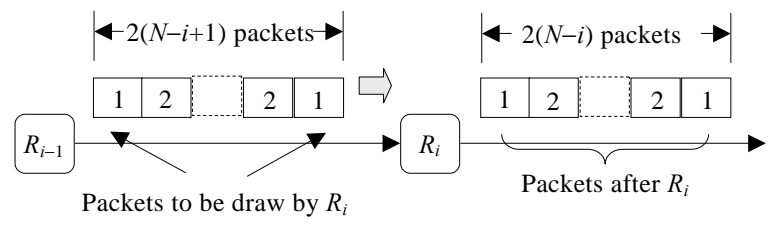

(b) The change of packet train at each node

(b) 数据包队列被中间节点改变的情况

Fig.12 BNeck probing technique

图 12 BNeck 的探测技术

该组探测数据包在 $L_{i}$ 上的传输间隔就等于首、尾两个 IP 包被 $R_{i+1}$ 接收的时间间隔 $\Omega_{i}$, 也即 $R_{i+1}$ 发送出两 个对应的 ICMP TE 包的间隔 $O_{i}$. 这两个 ICMP TE 包被 $R_{0}$ 接收的时间间隔是 $\Delta_{i}$, BNeck 假设 $\Omega_{i}=O_{i}=\Delta_{i}$.

首先, 相邻两个探测数据包在 $L_{i}$ 上真实的平均传输间隔(per-packet dispersion, 简称 PPD) 是

$$
q_{i}=\frac{\Omega_{i}}{2 \cdot(N-i)-1}(0 \leq i \leq n-1)
$$

其次,相应的两个 ICMP TE 包被 $R_{i}$ 发送出来的间隔是 $O_{i}$, 据此计算出来的 PPD 是 


$$
r_{i}=\frac{O_{i}}{2 \cdot(N-i)-1}(0 \leq i \leq n-1)
$$

最后, 这两个 ICMP TE 包抵达 $R_{0}$ 时的间隔(即被 $R_{0}$ 接收的间隔)为 $\Delta_{i}$, BNeck 根据 $\Delta_{i}$ 计算出来的 PPD 为

$$
p_{i}=\frac{\Delta}{2 \cdot(N-i)-1}(0 \leq i \leq n-1)
$$

这里, $q_{i}, r_{i}$ 和 $p_{i}$ 分别被称为真实 PPD、最佳估算 PPD 和估算 PPD.

BNeck 系统设计中非常关键的一点是它假设 $q_{i}=r_{i}=p_{i}, 0 \leq i \leq n-1$. 在一次探测之后,BNeck 收集了在 $x$ 探测速 率下的 PPD 集合 $\left\{p_{0}, p_{1}, \ldots, p_{n-1}\right\}$, 并分析该集合的变化规律, 进而估算 $x$ 与 $A_{P}$ 的关系,然后以不同的速率再探测再估算, 以至最后确定可用带宽.

现在用图 9 中的实验平台做一个简单的实验, 来说明反向路径中的背景数据流是如何影响或破坏上述假 设的.

我们在 $R_{0}$ 上安装 BNeck 系统,用以度量整个路径的可用带宽;同时,在 $R_{0}, \ldots, R_{6}$ 上运行 Iperf 以生成反向的 OHP 数据流, 并配置 Iperf 的参数让 $R_{i}$ 到 $R_{i-1}(1 \leq i \leq 6)$ 共 6 个反向链路的利用率都相同. 此外, 在 $R_{3}$ 和 $R_{4}$ 上运行 Iperf 以生成 $40 \mathrm{Mbps}$ 的 $R_{3}$ 到 $R_{4}$ 的 $\mathrm{OHP}$ 背景数据流. 那么, $A_{P}=60 \mathrm{Mbps}$ 且 $L_{3}$ 是瓶颈链路. 当反向路径中每个链路的利 用率为 $(0 \%, 20 \%, 50 \%)$ 时, 我们运行 BNeck 度量可用带宽并记录每次探测中的 $p_{5}$; 同时,在 $R_{6}$ 上安装 ethereal ${ }^{[34]}$ 以监听 $R_{6}$ 收发的所有 IP 包并计算 $r_{5}$. 实验结果如图 13 所示.

我们发现,随着链路利用率的上升, $p_{5}$ 与 $r_{5}$ 的差距也逐渐增大, 且 $p_{5}$ 的波动也增大. 这说明反向路径背景数据 流对应答数据包的传输有明显影响,ICMP TE 包在离开 $R_{6}$ 时的 PPD 间隔 $\left(r_{5}\right)$ 与其抵达 $R_{0}$ 时的 PPD 间隔 $\left(p_{5}\right)$ 差 异较大,这种差异将会导致度量准确度的下降.

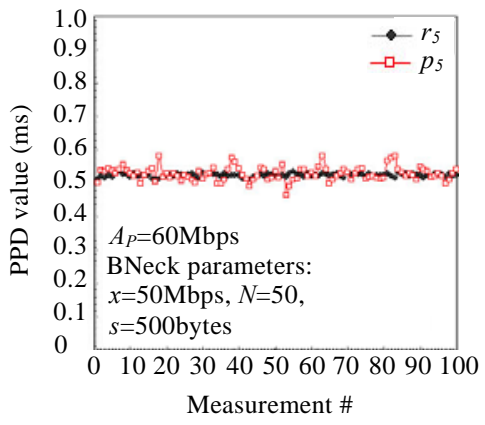

(a) All link utilization of reverse path is $0 \%$

(a) 所有反向链路的利用率为 $0 \%$

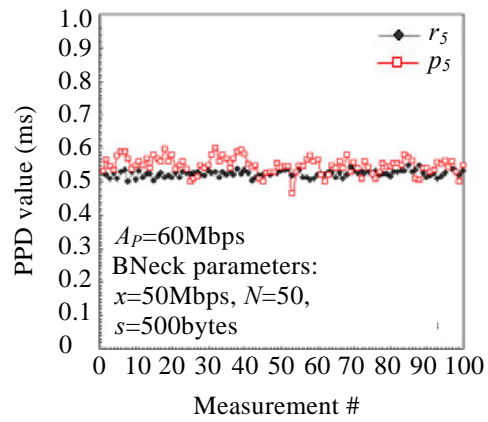

(b) All link utilization of reverse path is $20 \%$

(b) 所有反向链路的利用率为 $20 \%$

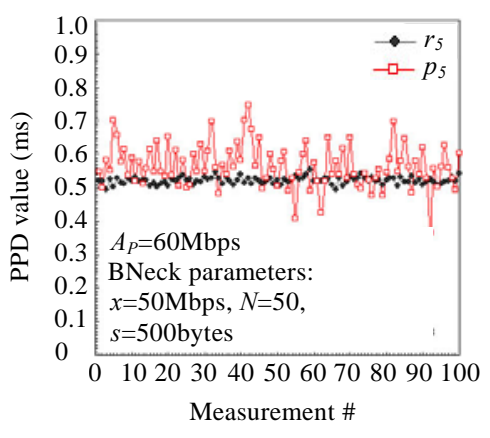

(c) All link utilization of reverse path is $50 \%$

(c) 所有反响链路的利用率为 $50 \%$

Fig.13 The impact of cross-traffic in reverse path on the PPD evaluation

图 13 反向路径中背景数据流对估算 PPD 的影响

\section{2 动态路由}

理论上,互联网中任意两节点间的路由都是动态可变的, 从一个节点发往另一个节点的多个数据包可能会 流经不同的网络路径.动态路由一方面是负载均衡的结果, 另一方面则是 ISP 传输策略和商业模式的产物 ${ }^{[35]}$. 研 究发现, 由两个 IP 地址 $a d d r_{-} a$ 和 $a d d r_{-} b$ 所限定的有向路径 $P: a d d r_{-} a \rightarrow a d d r_{-} b$ 在以小时为单位的时间内是基 本不变的 ${ }^{[36]}$. 这意味着, 在多个相邻的度量周期内, 从 $R_{0}$ 发往 $R_{n}$ 的探测数据包确实会依次流经相同的中间节点. 然而,进一步的研究 ${ }^{[18]}$ 也表明:

(1) $P_{1}: a d d r_{-} a \rightarrow a d d r \_b$ 与 $P_{2}: a d d r_{-} b \rightarrow a d d r_{-} a$ 有可能不相同,即 $P_{1}$ 和 $P_{2}$ 可能包含不同的中间节点. 以图 14 的实验网络为例, 从 $R_{0}$ 到 $R_{6}$ 的路径与从 $R_{6}$ 回到 $R_{0}$ 的一条路径(1)就不相同. 
（2）假设 $P: a d d r_{-} a \rightarrow a d d r_{-} b, a d d r_{-} c$ 和 $a d d r_{-} d$ 分别为 $P$ 的中间节点并且路径 $P_{A C}: a d d r_{-} a \rightarrow a d d r_{-} c$ 属于 $P_{A D}: a d d r \_a \rightarrow a d d r_{-} d$, 然而, 反向路径 $P_{C A}: a d d r_{-} c \rightarrow a d d r_{-} a$ 有可能不属于 $P_{D A}: a d d r_{-} d \rightarrow a d d r \_a$. 不少单 终端系统需要路径中每一个中间节点都应答,不同中间节点返回的应答数据包可能会走不同的路 由.比如图 14 中的 $R_{0}$ 向 $R_{3}$ 和 $R_{4}$ 分别发送探测数据包,然后它会收到 $R_{3}$ 和 $R_{4}$ 返回的应答数据包,从 $R_{3}$ 到 $R_{0}$ 的数据包所走的路径(2)就不属于从 $R_{4}$ 到 $R_{0}$ 的一条路径(3).

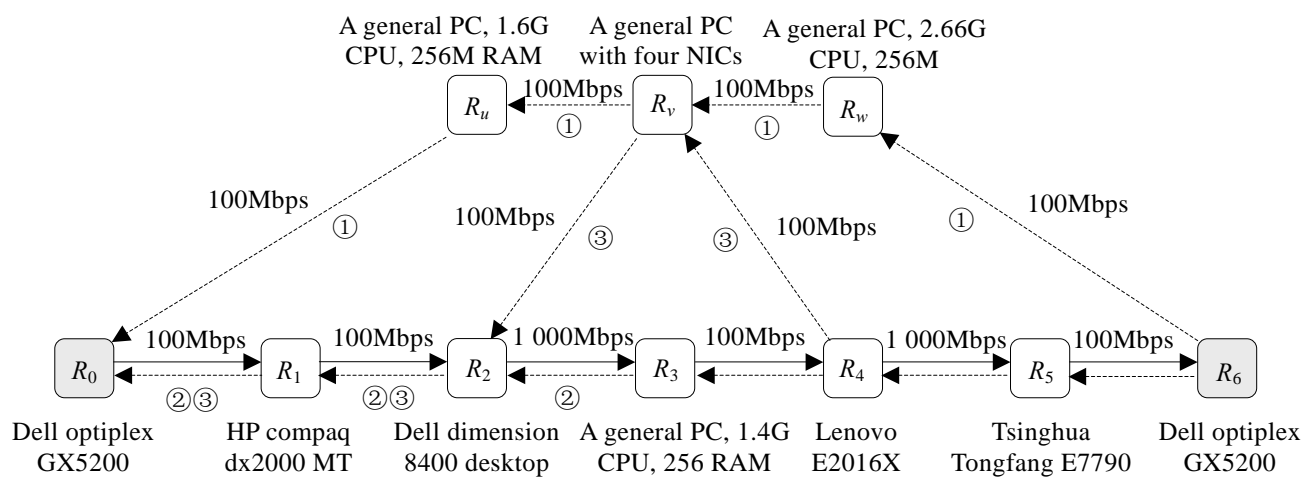

Fig.14 In-House experimental network with dynamic routes

图 14 存在动态路由的机构内部实验网络

路由的动态性和不确定性意味着反向路径的属性(比如带宽容量、可用带宽、拥塞情况和背景数据流等) 可能不是固定的,而是动态变化的.所以,反向路径中的动态路由将影响从中间节点 $R_{i}$ 向 $R_{0}$ 传输的应答数据包. 但问题是这种影响难以被量化描述,所以,大多数单终端系统都仅仅假设应答数据包在反向路径上受到的影响 微乎其微(或每一个应答数据包受到的影响都基本相同).

下面以 BNeck 系统的 PPD 估算为例,分析动态路由对应答数据包(乃至度量过程)的影响.

实验平台如图 14 所示,所有节点上都安装 Iperf,并配置 Iperf 以在 $L_{3}$ 中生成 $40 \mathrm{Mbps}$ 的 OHP 背景数据流; 同时,在其他所有链路中随机生成 $0 \mathrm{Mbps} \sim 30 \mathrm{Mbps}$ 的 OHP 背景数据流. 于是, $A_{P}=A_{3}=60 \mathrm{Mbps}$. 此外, 每个节点都被 设置为根据负载动态选择可用的路由, 那么, $R_{4}$ 到 $R_{0}$ 的路由共有 $R_{4}-R_{v}-R_{u}-R_{0}, R_{4}-R_{v}-R_{2}-R_{1}-R_{0}$ 和 $R_{4}-R_{3}-R_{2}-R_{1}-R_{0}$ 三 种可能;类似地, $R_{6}$ 到 $R_{0}$ 的路由存在 5 种可能.

实验分为逐渐复杂的 3 部分:

(a) 没有 $R_{u}, R_{v}$ 和 $R_{w}$. 此时, 前向路径与反向路径经过同一组路由器.

(b) 仅有 $R_{v}$, 没有 $R_{u}$ 和 $R_{w}$. 这时, $R_{4}, R_{5}$ 和 $R_{6}$ 到 $R_{0}$ 的反向路径都有两种选择(要么经过 $R_{v}$, 要么经过 $R_{3}$ ).

(c) $R_{u}, R_{v}$ 和 $R_{w}$ 都有.此时的反向路由是最复杂的,涵盖了图 14 中所有可能的情况.

在每一部分实验中, 都运行 BNeck 系统度量可用带宽 30 次, 结果如图 15 所示. 其中, 图 15(b)和图 15(c)两种 情况与图 15(a)相比,相同点是 $p_{1}, p_{2}$ 和 $p_{3}$ 都比较稳定, 区别是 $p_{4}$ 与 $p_{5}$ 起伏相当大,这正是动态路由所造成的. 图 15 还给出了 3 种情况下 BNeck 的可用带宽度量结果. 容易看出, 在(a)情况下,度量结果非常好地收敛在

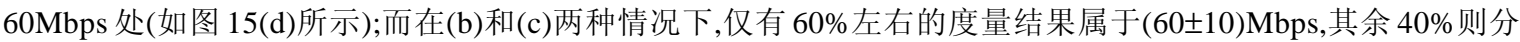
散在 20Mbps 50Mbps 和 70Mbps 85Mbps 的范围内(如图 15(e)、图 15(f)所示). 这说明动态路由明显影响了度 量结果的准确度,而且路由情况越复杂,度量结果的误差就越大. 


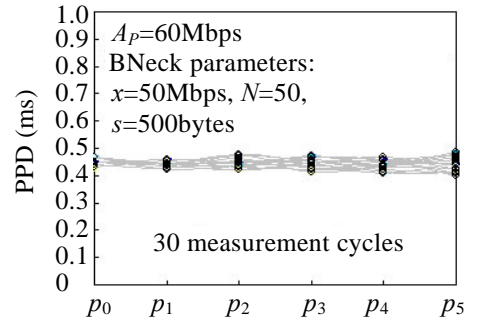

(a) The PPD of each link without

$$
R_{u}, R_{v} \text { or } R_{w}
$$

(a) 没有 $R_{u}, R_{v}$ 和 $R_{w}$ 时每个链路的 PPD

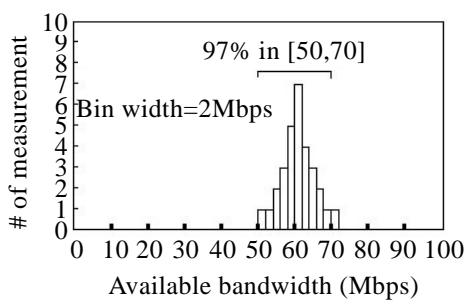

(d) The measurement result of BNeck under (a)

(d) 在(a)情况下 BNeck 的度量结果

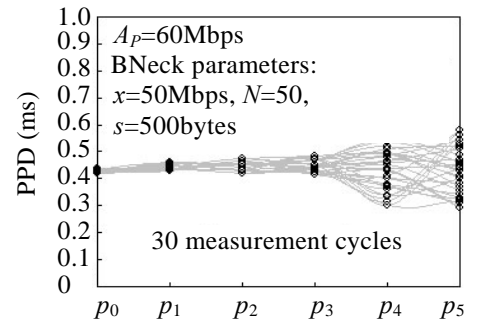

(b) The PPD of each link with $R_{v}$

(b) 仅有 $R_{v}$ 时每个链路的 PPD

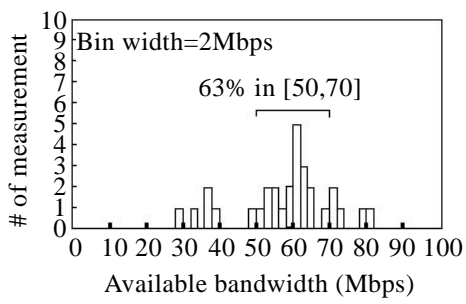

(e) The measurement result of BNeck under (b)

(e) 在(b)情况下 BNeck 的度量结果

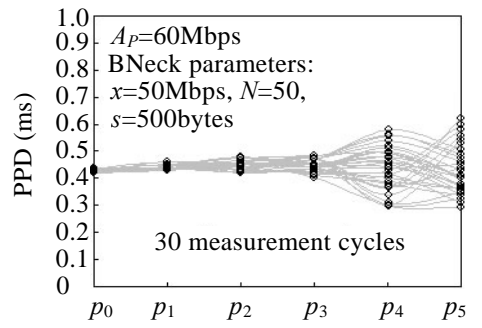

(c) The PPD of each link with $R_{u}, R_{v}$ and $R_{w}$

(c) 同时有 $R_{u}, R_{v}$ 和 $R_{w}$ 时每个链路的 PPD

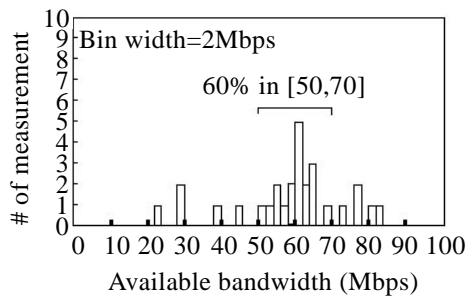

(f) The measurement result of BNeck under (c)

(f) 在(c)情况下 BNeck 的度量结果

Fig.15 PPD and measurement results of BNeck under different route conditions

图 15 在不同路由情况下 BNeck 的 PPD 估算和可用带宽度量结果

\subsection{ICMP处理时间}

为了度量可用带宽,大部分单终端系统需要收集和分析从待测量路径中返回的 ICMP 包.ICMP 处理时间指 的是某个节点从接收到能够触发 ICMP 处理流程的探测数据包起,到它生成对应的 ICMP 包并把该 ICMP 包发 送进传输媒介的时刻为止的一段时间. 研究表明 ${ }^{[37]}$,一个节点的 ICMP 处理时间是变化的,通常从几十微秒到几 百毫秒不等.但是, 由于目前尚没有办法从 $R_{0}$ 处准确获知远端节点的 ICMP 处理时间,所以,度量系统往往都默认 把 ICMP 处理时间忽略不计或等同于某个常数,下面的分析表明,这种做法存在三大问题:

首先,ICMP 处理时间是不可忽略的,且每个节点的 ICMP 处理时间也不是固定的.Paxson 于 2000 年运行 fsd 程序 ${ }^{[37]}$ 度量了数百个路由器的 ICMP TE 包的处理时间(如图 16(a)所示). 我们不加修改地采用同样的方法,在 表 4 的覆盖型实验网络平台上运行 fsd,结果如图 16(b)所示.我们发现,尽管 6 年来互联网经历了巨大的变化,但 ICMP TE 处理时间的分布仍然是基本一致的,且 $85 \%$ 以上的处理时间都集中在 0 1s 内. 除此之外,我们还测量了 单个节点的 ICMP 处理时间.将图 9 中的 $R_{1}$ 换成一台 Cisco 1800 系列路由器, 由 $R_{0}$ 向 $R_{1}$ 以恒定的速率(10 个 包/s)发送 ICMP echo-request 包,并通过 ethereal ${ }^{[34]}$ 监听 ICMP 包的发送与接收时刻进而计算出 $R_{1}$ 的 ICMP 处理 时间.结果如图 16(c)所示, 只有 96\%的 ICMP echo-request 包成功触发了 $R_{1}$ 的 ICMP 处理流程. 另外, 虽然大部分 ICMP 处理时间都分布在 $90 \mathrm{~ms}$ 100ms 内,但仍可以看出单个节点的 ICMP 处理时间是不断变化的,相邻处理时 间的最大差值为 $43 \mathrm{~ms}$.

其次,动态变化的 ICMP 处理时间会影响度量精度.这里仍然以 BNeck 系统为例来说明这一点,实验平台采 用图 9 的网络且设置与第 3.1 节中的实验相同(反向链路利用率均为 $20 \%$, 同时, $A_{P}=A_{3}=60 \mathrm{Mbps}$ ). 图 17(a)展示了 一次数据包探测过程中记录的 $q_{i}$ 和 $r_{i}$ 值 $(0 \leq i \leq 5), q_{i}$ 是指该组探测数据包在 $L_{i}$ 上的真实 $\mathrm{PPD}$, 而 $r_{i}$ 是根据 $R_{i+1}$ 生 成 ICMP TE 包的时间差计算出来的 PPD. 这两个 PPD 的曲线尽管很接近,但是仍有所区别,其区别就在于 $r_{i}$ 包 含了 ICMP 处理时间而 $q_{i}$ 没有.接下来将 BNeck 的核心算法直接用于 $q_{i}$ 和 $r_{i}$ 上(即在计算中用 $q_{i}$ 和 $r_{i}$ 分别替代 
$p_{i}$ ), 连续 30 次度量的结果如图 17(b)和图 17(c)所示. 我们发现, 虽然 BNeck 的度量结果都聚集在 60Mbps 处,但是 图 17(b)中有 90\%的结果在(60土10)Mbps 的范围内, 而图 17(c)中仅有 83\%. 可见,ICMP 处理时间确实会影响单终 端系统的度量精度.

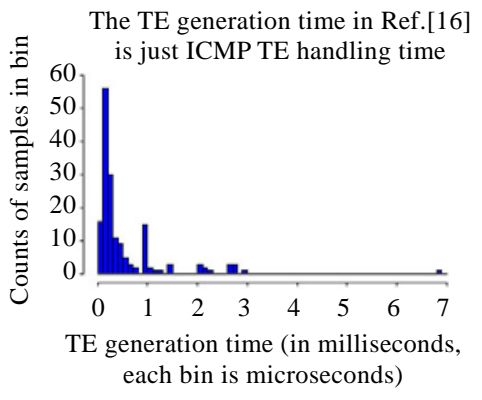

(a) Paxson's measurements in $2000^{[16]}$

(a) Paxson 在 2000 年的 度量结果 ${ }^{[16]}$

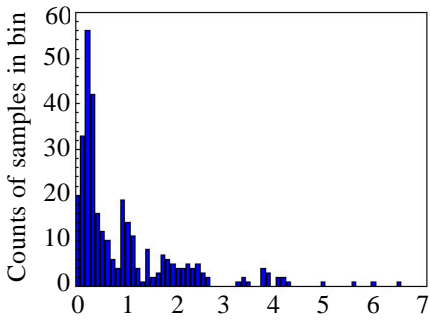

TE generation time (in milliseconds, each bin is microseconds)

(b) Our measurements on experimental overlay network in 2005

(b) 2005 年在实验性覆盖网络 上的度量结果

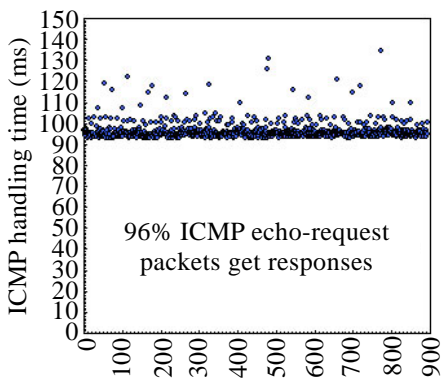

Number of ICMP echo-request probes

(c) Our measurements on a Cisco1800 series router

(c) Cisco1800 系列路由器 上的度量结果

Fig.16 Handling time of ICMP time-exceed (TE) error packets

图 16 ICMP TE(time-exceed)数据包的处理时间

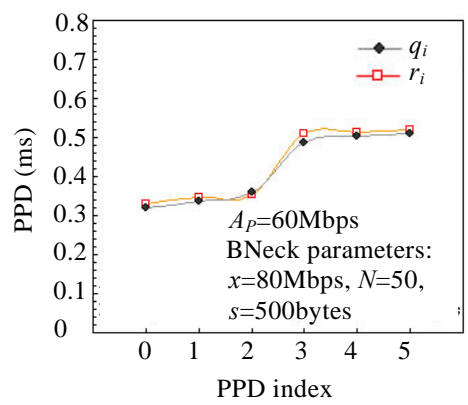

(a) Two PPD measurements in a probing process

(a) 在一次探测中的两类 PPD 度量结果

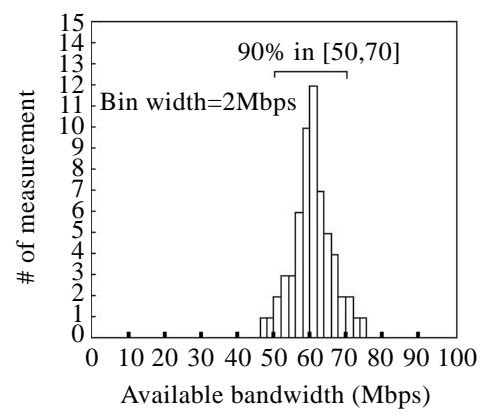

(b) The measurement result of BNeck using $q_{i}$

(b) 使用 $q_{i}$ 时 BNeck 的度量结果

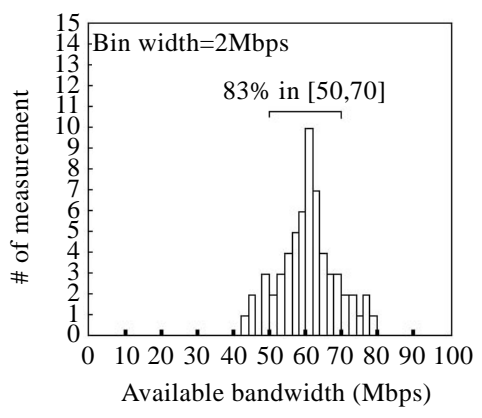

(c) The measurement result of BNeck using $r_{i}$

(c) 使用 $r_{i}$ 时 BNeck 的度量结果

Fig.17 ICMP handling time of a normal PC and a router (10 ICMP echo-request per second)

图 17 普通 PC 和路由器的 ICMP 处理时间(每秒发送 10 个 ICMP echo-request 包)

最后, 与 ICMP 处理时间相关的一个重要问题是 ICMP 限速. 近 5 年来, 路由器逐渐开始限制 ICMP 数据包 的产生速率.如果相邻探测数据包的发送间隔较小,那么,部分探测数据包就很可能无法触发路由器的 ICMP 应 答.例如,很多 Cisco 路由器在一段固定的时间内(如 1s) 就仅发送一个 ICMP 包 ${ }^{[37]}$. 这样做的好处是路由器能够更 全面地抵抗拒绝服务攻击, 并且避免网络资源被恶意使用(ICMP 也是一种资源) ${ }^{[38]}$. 所以,如果单终端度量系统 不能及时觉察到路由器的 ICMP 速率限制并采取相应措施, 它就无法正确地度量可用带宽. 比如, 它会误以为未 收到 ICMP 应答包是因为对应的探测数据包在传输过程中丢失了, 并把包丢失的原因归结为发送速率高于可用 带宽而造成的网络拥塞,但事实却并非如此.另外,因为 ICMP 速率限制是可以由路由器根据情况定制的,不同路 由器的 ICMP 产生速率可能会有所不同. 为了更有效地使用 echo 技术并正确判断探测数据包与应答数据包的 一一对应关系,单终端系统需要获知待测路径中各个节点的 ICMP 速率限制情况. 然而, 问题是目前尚没有较为 可行的技术能够做到这一点. 


\section{4 双终端系统独有的基本问题}

与单终端系统相比,双终端系统基本问题较少,而且度量结果也较为准确.其原因是双终端系统充分利用 $R_{0}$ 和 $R_{n}$ 之间的协作简化了度量过程,而不像单终端系统那样必须面对反向路径的干扰. 然而, 双终端系统所独有的 两大基本问题(软件安装和时钟同步),正是来源于它对 $R_{0}$ 和 $R_{n}$ 的依赖.

\section{1 软件安装}

软件安装是双终端系统独有的基本问题.双终端系统要求在待测网络路径的首、尾节点 $R_{0}$ 和 $R_{n}$ 上都安装 相应的软件, 这一要求给用户带来了很大的麻烦. 在通常情况下, 用户只能在自己的机器上安装和运行软件, 而 没有权限在网络路径另一端的主机上做同样的事情.但如果待测路径的两终端没有同时安装相应的软件, 那么 该路径的可用带宽就无法被度量.所以,软件安装问题严重限制了双终端系统的应用和推广.与此相反, 单终端 系统只需安装在 $R_{0}$ 上就能用来度量以 $R_{0}$ 为源终端主机的大多数网络路径(在实际应用中, 部分网络路径因为不 支持 echo 技术,所以它们将无法被度量).

从另一个角度来讲, 双终端系统在每个节点上的安装都非常宝贵. 人们通常在覆盖型网络上部署双终端度 量系统, 以求充分利用已经安装的双终端软件.一个覆盖型网络由几十个(或几百个)分布在不同地理位置的主 机联结而成, 典型的覆盖型网络一般都部署在校园网内.近年来,跨地域的覆盖型实验网络也获得了长足的发 展, 较为知名的有 $\mathrm{MINI}^{[39]}$ 和 PlanetLab ${ }^{[40]}$ 等. 采用覆盖型网络的好处是通过协调网络中的 $k$ 个节点, 我们最多可 以度量 $k(k-1)$ 条有向网络路径. 举例来说,通过在 PlanetLab 中的约 500 个节点上统一部署双终端度量系统, 我们 可以度量近 250000 条网络路径的可用带宽, 这是一个非常可观的数字, 对于扩大双终端系统的适用范围帮助 很大. 然而, 覆盖型网络也要面对两个关键问题: 首先, 维护大规模的覆盖型网络往往是一个非常复杂的系统工 程, 它需要综合考虑多方面的问题(如软件的统一安装、卸载与升级、应用程序的系统兼容性以及用户的授权 认证等等 ${ }^{[39]}$; 其次, 如果一个待测网络路径的源主机或目标主机并不在覆盖型网络中, 那么, 双终端系统还是无 法度量该路径的可用带宽.

\section{2 时钟同步}

时钟同步是双终端系统独有的另一基本问题.通常情况下,源主机 $R_{0}$ 和目标主机 $R_{n}$ 分别记录探测数据包的 发送时间和接收时间,并分别维护时间集合 $T_{S}$ 和 $T_{R}$. 但问题在于, $R_{0}$ 和 $R_{n}$ 用于记录时间的系统时钟可能是不同 步的.从度量系统的角度看,时钟不同步有 3 种含义:首先是绝对时间不相同,对于那些依靠绝对时间而不是时间 间隔来计时的双终端系统而言(如 Delphi 系统的部分计时过程就依赖于两端主机的绝对时间),如果两端主机的 时钟不同(比如一个是 1:00 AM, 而另一个是 1:10 AM), 这就会影响到度量结果的正确性; 第二是时钟的突发调整 (abrupt adjustment) ${ }^{[24]}$,一端时钟的突发调整会让两个原本一致的时钟突然变得不同步,进而打乱度量系统的线 性计时过程; 第三是更新频率不相同,时钟更新速率是指在现实世界的 $1 \mathrm{~s}$ 内,计算机时钟前进的时间计数(有可 能不是 $1 \mathrm{~s}$, 而是在 $1 \pm 10^{-5} \mathrm{~s}$ 范围内的某个值). 更新频率的不同是导致度量精度下降的一大原因. 举例来说, 当运行 Pathload 系统时, 如果 $R_{n}$ 的时钟更新速率比 $R_{0}$ 快 $10 \%$, 那么,即使探测速率低于可用带宽并且数据包队列在传输 途中没有遇到任何网络拥塞, $R_{n}$ 计算出来的数据包间隔也可能会呈现出递增趋势. 正是这样一个呈递增趋势的 信息,让 Pathload 错误地认为探测速率高于可用带宽,进而误导了后续的所有探测.

现以实验来说明时钟不同步对度量过程的严重影响.仍以图 9 中的线性网络作为实验平台,在 $R_{0}$ 和 $R_{6}$ 上分 别安装 Pathload 的发送端 pathload_snd 和接收端 pathload_rcv, 且记录下探测数据包的相关单路延迟(relative one-way-delay, 简称 ROWD). 分析 ROWD 的变化趋势, 并据此判断探测速率与可用带宽的关系是 Pathload 的核 心内容.容易看出,当探测速率约等于可用带宽时,ROWD 没有明显的变化趋势(如图 18(a)所示).接下来假设 $R_{6}$ 的时钟更新速率比 $R_{0}$ 快 10\%,那么, 记录下来的 ROWD 如图 18(b)所示, 这意味着同一次探测在不同的时钟更新 速率下,会有截然不同的估算结果. 时钟更新频率不同所导致的后果是:Pathload 在正常情况下以及在 $R_{6}$ 的时钟 更新频率比 $R_{0}$ 快 $10 \%$ 的情况下, 输出的度量结果(带宽区间)有很大差别, 且这种差别还会随着 $A_{P}$ 的不同而变化 (如图 18(c)所示).总之, 当 $R_{0}$ 与 $R_{n}$ 的时钟更新频率不同时,Pathload 输出的带宽区间是非常不准确的, 它往往不 
包括 $A_{P}$.

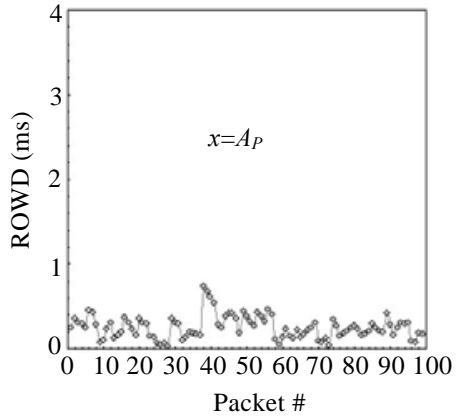

(a) The clock rates of $R_{0}$ and $R_{n}$ are synchronous

(a) $R_{0}$ 和 $R_{n}$ 的时钟频率同步时

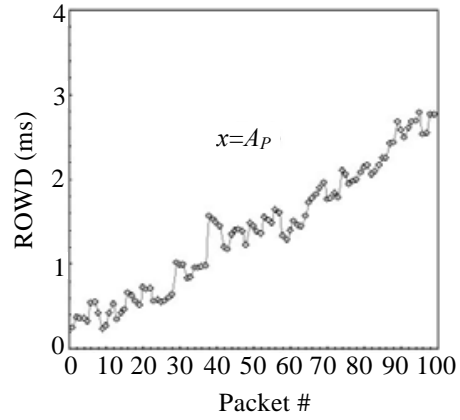

(b) The clock rates of $R_{n}$ is $10 \%$ quicker than that of $R_{0}$

(b) $R_{n}$ 的时钟频率比 $R_{0}$ 快 $10 \%$ 时

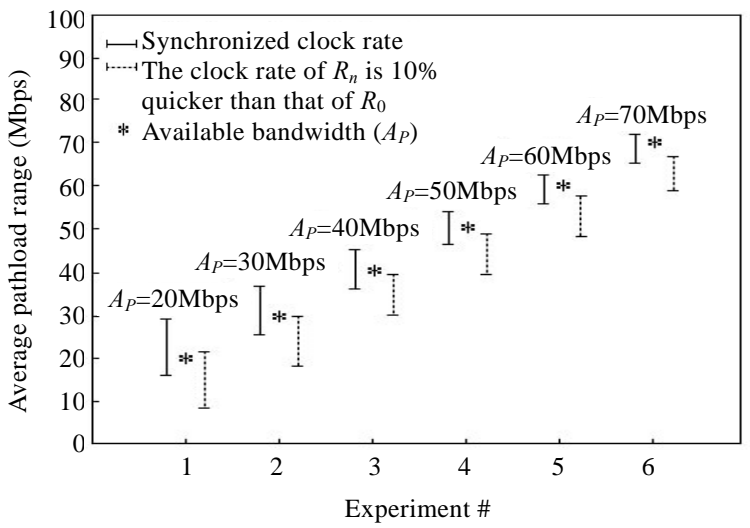

(c) The measurement result of Pathload under (a) and (b)

(c) 在(a)和(b)两种情况下,Pathload 的度量结果

Fig.18 The impact of different clock rates on measurement result

图 18 不同时钟更新速率对度量结果的影响

为了同步 $R_{0}$ 和 $R_{n}$ 的时钟,研究者们已经做了大量的工作, 然而, 已有的成果只能保证时钟在以分或小时为 单位的时间内是同步的,还无法做到更细粒度的同步 ${ }^{[24]}$. 互联网是如此庞大复杂,充满了大量异构的系统, 要想 在两台远程主机之间做到完全的时钟同步是相当困难的.时钟同步问题不仅在带宽度量系统中存在,而且在很 多领域中也同样存在 ${ }^{[24]}$.

\section{5 结束语}

本文分析了 13 个常见的度量系统,并在理论分析和实验数据的基础上深入研究了这些系统中普遍存在的 基本问题.研究中采用的是对这 13 个系统都适用的理论模型和分析方法,并且使用大量有针对性的实验数据. 这些数据来源于多种实验平台,包括不同软、硬件配置的单个节点,机构内部搭建的实验网络以及跨地域的大 规模覆盖型网络等.研究表明,正是这些基本问题导致了度量结果的高度不确定性,从而极大地限制了度量系统 的适用范围.深入研究这些基本问题并寻求解决方法, 不仅有利于设计和开发出在真实网络环境中高效运行的 度量系统,而且有利于从整体上推动网络度量技术的发展.

此外, 在研究基本问题的过程中, 我们开展了富有成效的网络实验, 不仅获得了大量宝贵的数据, 而且在数 据分析的基础上总结出不少有价值的观点.例如,批量 TCP 吞吐量并不等于可用带宽,且使用多个并发的 TCP 
连接比扩大 TCP 缓存窗口的方法更能获得额外的 TCP 吞吐量; 又如,系统调用延迟和 ICMP 处理时间对任意一 个节点而言都不是固定的数值, 而是随着软、硬件平台和系统负载的不同而变化;再如,数据包的异常传输是互 联网中的普遍现象, 其中以包丢失和乱序传送最为明显.这些从大量实验数据中分析得来的结论有助于我们进 一步加深对可用带宽及其度量过程的理解.

\section{References:}

[1] Jain M, Dovrolis C. End-to-End available bandwidth: Measurement methodology, dynamics, and relation with TCP throughput. IEEE/ACM Trans. on Networking, 2003,11(4):537-549.

[2] Jacobson V. Congestion avoidance and control. ACM SIGCOMM Computer Communication Review, 1995,25(1):157-187.

[3] Li D, Wu JP, Cui Y, Xu L, Liu Y. Modeling and improvement of PIM-SM protocol. Journal of Software, 2006,17(2):285-294 (in Chinese with English abstract). http://www.jos.org.cn/1000-9825/17/285.htm

[4] Liu M, Li ZC, Guo XB, Deng H. An end-to-end available bandwidth estimation methodology. Journal of Software, 2006,17(1): 108-116 (in Chinese with English abstract). http://www.jos.org.cn/1000-9825/17/108.htm

[5] Zhou H, Wang YJ, Wang Q. Measuring Internet bottlenecks: Location, capacity, and available bandwidth. In: Proc. of the Int'l Conf. on Computer Network and Mobile Computing. New York: Springer-Verlag, 2005. 1052-1062.

[6] Hu NN, Steenkiste P. Evaluation and characterization of available bandwidth probing techniques. IEEE Journal on Selected Areas in Communications, 2003,21(6):879-894.

[7] Antoniades D, Athanatos M, Papadogiannakis A, Markatos E, Dovrolis C. Available bandwidth measurement as simple as running wget. In: Proc. of the Passive and Active Measurement Conf. 2006. http://www.ics.forth.gr/dcs/Activities/papers/abget.pdf

[8] Akella A, Seshan S, Shaikh A. An empirical evaluation of wide-area Internet bottlenecks. In: Proc. of the ACM SIGCOMM Conf. on Internet Measurement. New York: ACM, 2003. 316-317.

[9] Carter R, Crovella M. Measuring bottleneck link speed in packet-switched networks. Performance Evaluation, 1996,27(28): 297-318.

[10] Ribeiro V, Coates M, Riedi R, Sarvotham S, Hendricks B, Baraniuk R. Multifractal cross-traffic estimation. In: Proc. of the ITC Conf. on IP Traffic, Modeling and Management. 2000. http://cmc.rice.edu/docs/docinfo.aspx?doc=Rib2000Sep5Multifract

[11] Netest and pipechar. 2005. http://dsd.lbl.gov/OldProjects/NCS/

[12] Ribeiro V, Riedi R, Baraniuk R, Navaratil J, Cottrell L. PathChip: Efficient available bandwidth estimation for network paths. In: Proc. of the Passive and Active Measurement Workshop. 2003. http://moat.nlanr.net/PAM2003/PAM2003papers/3824.pdf

[13] Saroiu S, Gummadi P, Gribble S. SProbe: A fast technique for measuring bottleneck bandwidth in uncooperative environments. In: Proc. of the IEEE INFOCOM. 2002. http://sprobe.cs.washington.edu/sprobe.ps

[14] Strauss J, Katabi D, Kaashoek F. A measurement study of available bandwidth estimation tools. In: Proc. of the ACM SIGCOMM Conf. on Internet Measurement. New York: ACM, 2003. 39-44.

[15] Melander B, Bjorkman M, Gunningberg P. A new end-to-end probing and analysis method for estimating bandwidth bottlenecks. In: Proc. of the IEEE Global Internet Symp. Piscataway: IEEE, 2000. 415-420.

[16] Postel J. Internet control message protocol. IETF RFC 792, 1981. http://www.faqs.org/rfcs/rfc792.html

[17] Dovrolis C, Ramanathan P, Moore D. Packet dispersion techniques and a capacity estimation methodology. IEEE/ACM Trans. on Networking. 2004,12(6):963-977.

[18] Paxson V. Measurements and analysis of end-to-end Internet dynamics [Ph.D. Thesis]. Berkeley: University of California, 1997.

[19] Network simulator (version 2). 1998. http://www.isi.edu/nsnam/ns/

[20] Jin G, Tierney B. System capability effects on algorithms for network bandwidth measurement. In: Proc. of the ACM SIGCOMM Conf. on Internet Measurement. New York: ACM, 2003. 27-38.

[21] Jain M, Dovrolis C. Ten fallacies and pitfalls on end-to-end available bandwidth estimation. In: Proc. of the ACM SIGCOMM Conf. on Internet Measurement. New York: ACM, 2004. 272-277.

[22] Lakshminarayanan K, Padmanabhan V, Padhye J. Bandwidth estimation in broadband access networks. In: Proc. of the ACM SIGCOMM Conf. on Internet Measurement. New York: ACM, 2004. 314-321.

[23] Zhou H, Wang YJ, Wang XL, Huai XY. Difficulties in estimating available bandwidth. In: Proc. of the IEEE Int'l Conf. on 
Communications. 2006. http://www.icc2006.org/index/confrence-program.html

[24] Mills D. Improved algorithms for synchronizing computer network clocks. IEEE/ACM Trans. on Networking, 1995,3(3):245-254.

[25] Prasad R, Jain M, Dovrolis C. Effects of interrupt coalescence on network measurements. In: Proc. of the Passive and Active Measurement Workshop. New York: ACM, 2004. 247-256.

[26] Iannaccone G, Diot C, Graham I, McKeown N. Monitoring very high speed links. In: Proc. of the ACM SIGCOMM Conf. on Internet Measurement. New York: ACM, 2001. 267-271.

[27] Traceroute. 1996. ftp://ftp.ee.lbl.gov/traceroute.tar.z

[28] Wang XX, Wang YJ, Zhou JH, Wang XL. Congestion control algorithm based on improved model in large-delay networks. Acta Electronica Sinica, 2005,33(5):842-846 (in Chinese with English abstract).

[29] Floyd S, Jacobson V. Random early detection gateways for congestion avoidance. IEEE/ACM Trans. on Networking, 1993,1(4): 397-413.

[30] Iperf. 1999. http://dast.nlanr.net/projects/iperf/

[31] Multi router traffic grapher (MRTG). 1995. http://oss.oetiker.ch/mrtg/

[32] Floyd S, Paxson V. Difficulties in simulating the Internet. IEEE/ACM Trans. on Networking, 2001,9(4):392-403.

[33] NIST net. 1997. http://snad.ncsl.nist.gov/itg/nistnet/

[34] Ethereal. 1999. http://www.ethereal.com/

[35] Tangmunarunkit H, Govindan R, Shenker S, Estin D. The impact of policy on Internet paths. In: Proc. of the IEEE INFOCOM. 2001. http://www.routeviews.org/papers/tangmunarunkit01impact.pdf

[36] Zhang Y, Duffield N, Paxson V, Shenker S. On the constancy of Internet path properties. In: Proc. of the ACM SIGCOMM Conf. on Internet Measurement. New York: ACM, 2001. 197-211.

[37] Govindan R, Paxson V. Estimating router ICMP generation delays. In: Proc. of the Passive and Active Measurements Workshop. 2002. http://www.icir.org/vern/papers/fsd-pam-02.pdf

[38] Baker F. Requirements for IP version 4 routers. IETF RFC 1812, 1995. http://www.faqs.org/rfcs/rfc1812.html

[39] Paxson V, Mahdavi J, Adams A, Mathis M. An architecture for large-scale Internet measurement. IEEE Communications, 1998, 36(8):48-54.

[40] PlanetLab. 2002. http://www.planet-lab.org/

\section{附中文参考文献:}

[3] 李丹,吴建平,崔勇,徐恪,刘荣.PIM-SM 协议的建模与改进.软件学报,2006,17(2):285-294. http://www.jos.org.cn/1000-9825/17/ 285.htm

[4] 刘敏,李忠诚,过晓冰,邓辉.端到端的可用带宽测量方法.软件学报,2006,17(1):108-116. http://www.jos.org.cn/1000-9825/17/ 108.htm

[28] 王晓䂀,王永吉,周津慧,王秀利.基于改进网络模型的大时滞网络拥塞控制算法.电子学报,2005,33(5):842-846.

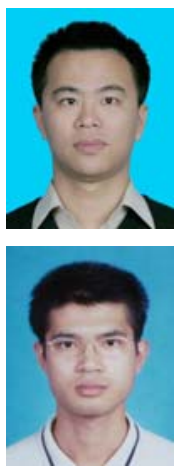

周辉 $(1980-)$, 男, 海南海口人, 博士, CCF 学生会员,主要研究领域为高可信网络系 统,网络性能,协同优化.

李丹(1981-), 男, 博士, 主要研究领域为计 算机网络体系结构, 组播, P2P,Overlay 网络.

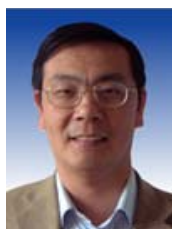

王永吉(1962-), 男, 博士, 研究员, 博士生 导师, 主要研究领域为计算机网络, 性能度 量, 实时系统。 